\title{
Transcriptome Analysis of the Barley-Deoxynivalenol Interaction: Evidence for a Role of Glutathione in Deoxynivalenol Detoxification
}

\author{
Stephanie A. Gardiner, ${ }^{1}$ Jayanand Boddu, ${ }^{1}$ Franz Berthiller, ${ }^{2}$ Christian Hametner, ${ }^{3}$ Robert M. Stupar, ${ }^{1}$ \\ Gerhard Adam, ${ }^{4}$ and Gary J. Muehlbauer ${ }^{1}$ \\ ${ }^{1}$ Department of Agronomy and Plant Genetics, University of Minnesota, St. Paul 55108, U.S.A.; ${ }^{2}$ Center for Analytical \\ Chemistry, Department of Agrobiotechnology (IFA-Tulln), University of Natural Resources and Applied Life Sciences, \\ Vienna; ${ }^{3}$ Institute for Applied Synthetic Chemistry, Vienna University of Technology, Vienna; ${ }^{4}$ Department of Applied \\ Genetics and Cell Biology, University of Natural Resources and Applied Life Sciences, Vienna
}

Submitted 31 October 2009. Accepted 9 March 2010.

Trichothecenes are a major group of toxins produced by phytopathogenic fungi, including Fusarium graminearum. Trichothecenes inhibit protein synthesis in eukaryotic cells and are toxicologically relevant mycotoxins for humans and animals. Because they promote plant disease, the role of host responses to trichothecene accumulation is considered to be an important aspect of plant defense and resistance to fungal infection. Our overall objective was to examine the barley response to application of the type $B$ trichothecene deoxynivalenol (DON). We found that DON is diluted by movement from the application site to acropetal and basipetal florets. A susceptible barley genotype converted DON to DON-3-O-glucoside, indicating that UDP-glucosyltransferases capable of detoxifying DON must exist in barley. RNA profiling of DON-treated barley spikes revealed strong upregulation of gene transcripts encoding ABC transporters, UDP-glucosyltransferases, cytochrome $P 450$ s, and glutathione- $S$-transferases. We noted that transcripts encoding cysteine synthases were dramatically induced by DON, and that toxin-sensitive yeast on glutathione- or cysteine-supplemented media or carrying a gene that encodes a cysteine biosynthetic enzyme exhibit DON resistance, suggesting that preventing glutathione depletion by increasing cysteine supply could play a role in ameliorating the impact of DON. Evidence for nonenzymatic formation of DON-glutathione adducts in vitro was found using both liquid chromatography-mass spectrometry and nuclear magnetic resonance analysis, indicating that the formation of DON-glutathione conjugates in vivo may reduce the impact of trichothecenes. Our results indicate that barley exhibits multiple defense mechanisms against trichothecenes.

Trichothecenes are a major group of mycotoxins that are produced by various species of Fusarium, Myrotherium, Trichoderma, Trichothecium, and Stachybotrys (Grove 2007). Most of the trichothecene producers are plant pathogens. Trichothecenes are structurally defined by an epoxide containing ses-

Corresponding author: G. J. Muehlbauer; Telephone: 612-625-6228; Fax: 612-625-1268; E-mail: muehl003@umn.edu

* The $\boldsymbol{e}$-Xtra logo stands for "electronic extra" and indicates that five supplementary tables and one supplementary figure are published online. quiterpenoid skeleton, and are further classified by the presence or absence of specific carbonyl groups. Two major classes of nonmacrocylic trichothecenes are distinguished by modification at the $\mathrm{C}_{8}$ position. Type $\mathrm{B}$ trichothecenes (e.g., nivalenol and deoxynivalenol [DON]) carry a keto group at $\mathrm{C}_{8}$, which is absent or replaced by (esterified) hydroxy groups in type A trichothecenes (T-2 toxin, HT-2 toxin, and 4,15-diacetoxyscirpenol) (Desjardins et al. 1993). Trichothecenes inhibit protein synthesis in eukaryotic cells and seem to function as virulence factors in the infection of plants (Desjardins et al. 2000). Therefore, plant host resistance to trichothecenes is expected to play an important role in resistance to toxin-producing fungal species.

Fusarium head blight (FHB), or head scab, is one of the most destructive diseases of wheat (Triticum aestivum L.) and barley (Hordeum vulgare L.) worldwide. FHB is caused primarily by the fungal pathogen Fusarium graminearum Schwabe (teleomorph = Gibberella zeae (Schwein.) Petch). The fungus infects spikes and produces the type B trichothecenes DON, 3-acetyl-deoxynivalenol (3-ADON), and 15-acetyl-deoxynivalenol (15-ADON), resulting in a reduction in yield and quality of the grain (Bai and Shaner 2004). The accumulation of DON and DON conjugates (Berthiller et al. 2005,2009 ) in grain also presents a serious health risk for both animals and humans (Pestka and Smolinski 2005).

The role that trichothecenes play during Fusarium spp. infection has been an active area of study. Immunocytochemical localization of trichothecene toxins in F. culmorum-infected wheat spikes detected toxin accumulation in the cytoplasm, chloroplast, plasmalemma, cell wall, and vacuole (Kang and Buchenauer 1999). Toxins were present in xylem vessels and phloem sieve tubes 4 to 6 days after inoculation, suggesting that the toxins can be readily translocated through the plant and, therefore, facilitate the development of disease. Accumulation of the toxins was also found to be closely associated with pathogenic symptoms in host cells (Kang and Buchenauer 1999). In addition, a variety of genetic studies have shown that trichothecene accumulation is associated with increased virulence of $F$. graminearum during wheat infection. Loss-of-function mutations in the $F$. graminearum TRI5 gene encoding the first enzyme (trichodiene synthase) in the trichothecene biosynthetic pathway resulted in fungal strains that do not produce trichothecenes and exhibited reduced virulence on wheat and barley (Proctor et al. 1995; Desjardins et al. 1996; Maier et al. 2006; Boddu et al. 2007). In wheat, trichothecenes were 
not required for initial infection but for the spread of disease (Bai et al. 2001).

A variety of genetic and biochemical studies have revealed mechanisms that may provide resistance to DON accumulation. In wheat, a major quantitative trait locus for FHB resistance was identified on the short arm of chromosome $3 \mathrm{~B}$. The region was originally designated as $Q f h s . n d s u-3 B S$ (Waldron et al. 1999) and was later designated as Fhbl (Liu et al. 2006). Wheat lines segregating for Fhbl exhibited an increased DON-glucoside/DON ratio after treatment with DON, implying that the metabolism of DON had a significant role in the FHB resistance complex (Lemmens et al. 2005). The authors proposed that Fhbl may encode a member of the UDP-glucosyltransferase gene family, or a regulator of a UDP-glucosyltransferase. A UDP-glucosyltransferase (DOGT1) gene capable of detoxifying DON into DON-3-Oglucoside (D3G) was identified in Arabidopsis thaliana (Poppenberger et al. 2003). Overexpression of the DOGT1 gene in Arabidopsis resulted in increased resistance of seedlings to DON (Poppenberger et al. 2003). Biochemical analysis also demonstrated that D3G does not inhibit in vitro translation of wheat ribosomes. In a study that measured D3G concentration in both DON- and $F$. graminearumtreated wheat plants, D3G levels were observed at 14 to $29 \%$ of the total DON concentration (Berthiller et al. 2005). In addition, wheat and maize naturally infected with Fusarium spp. were found to contain a proportion of D3G to DON of up to $70 \%$ (corresponding to $46 \mathrm{~mol} \%$ ) (Berthiller et al. 2009). Thus, it is apparent that gaining an understanding of the biochemical fate of DON is an important aspect in understanding mechanisms of host defense.

A variety of studies have been conducted to examine host responses to trichothecene accumulation. Transcript accumulation was examined in barley inoculated with either a wild-type F. graminearum strain (trichothecene producing) or a gene disruption mutant (tri5, trichothecene nonproducing), or mock water treated (Boddu et al. 2007). Two contrasting host responses were identified, including i) accumulation of gene transcripts encoding potentially trichothecene-detoxifying and transport proteins that likely protect the plant during infection and ii) accumulation of gene transcripts that encode cell deathand ubiquitination-related proteins that may result in increased susceptibility. Microarray analysis conducted with T-2 toxintreated $A$. thaliana illustrated induction of elicitor-like activity of this type A trichothecene. Of particular interest is the Arabidopsis gene, AtNFXL1, a homologue of the human putative transcription repressor NF-X1, which appears to act as a negative regulator of trichothecene defense responses (Asano et al. 2007). In DON-treated wheat plants, Desmond and associates (2008) observed hallmarks of cell-death activity, including the induction of reactive oxygen species and DNA laddering. In contrast, Walter and associates (2008) observed that DON-induced genes encoding proteins involved in alleviating cellular damage to the host plant. Lulin and associates (2010) treated wheat with DON and identified a set of glucosyltransferase gene transcripts with increased transcript accumulation. Taken together, these results indicate that trichothecenes, depending on the concentration studied and the host genotype, are capable of inducing host mechanisms that provide plant defense, or inducing negative regulator genes or cell-death genes that increase plant susceptibility to pathogen infection.

In this study, our overall objective was to develop an understanding of the host response in barley to DON. The specific objectives of this study were to i) examine the fate of DON in barley, ii) examine transcript accumulation patterns in barley in response to DON application, iii) examine the fate of DON in relation to the DON-induced transcript accumulation patterns, iv) identify key genes potentially involved in host defense to DON, and v) examine the formation of DON-glutathione (GSH) conjugates.

\section{RESULTS}

Disappearance of DON and formation of D3G in barley.

To examine the fate of DON in barley, we inoculated barley florets with DON two to three days after spike emergence. DON $(2 \mu \mathrm{g}$ or $6.75 \mathrm{nmol})$ dissolved in $10 \mu \mathrm{l}$ of water $(200$ ppm) was directly applied between the palea and lemma of nine florets in the middle of the spike (Supplementary Fig. 1A). DON concentration of inoculated floret tissue was measured at six timepoints: 1, 6, 12, 24, 48, and $72 \mathrm{~h}$ after inoculation (hai). In the inoculated florets, DON concentration followed an overall decreasing trend across the six timepoints, ranging from 6.22 to $0.90 \mathrm{nmol}$ (Fig. 1). Noninoculated florets imme-

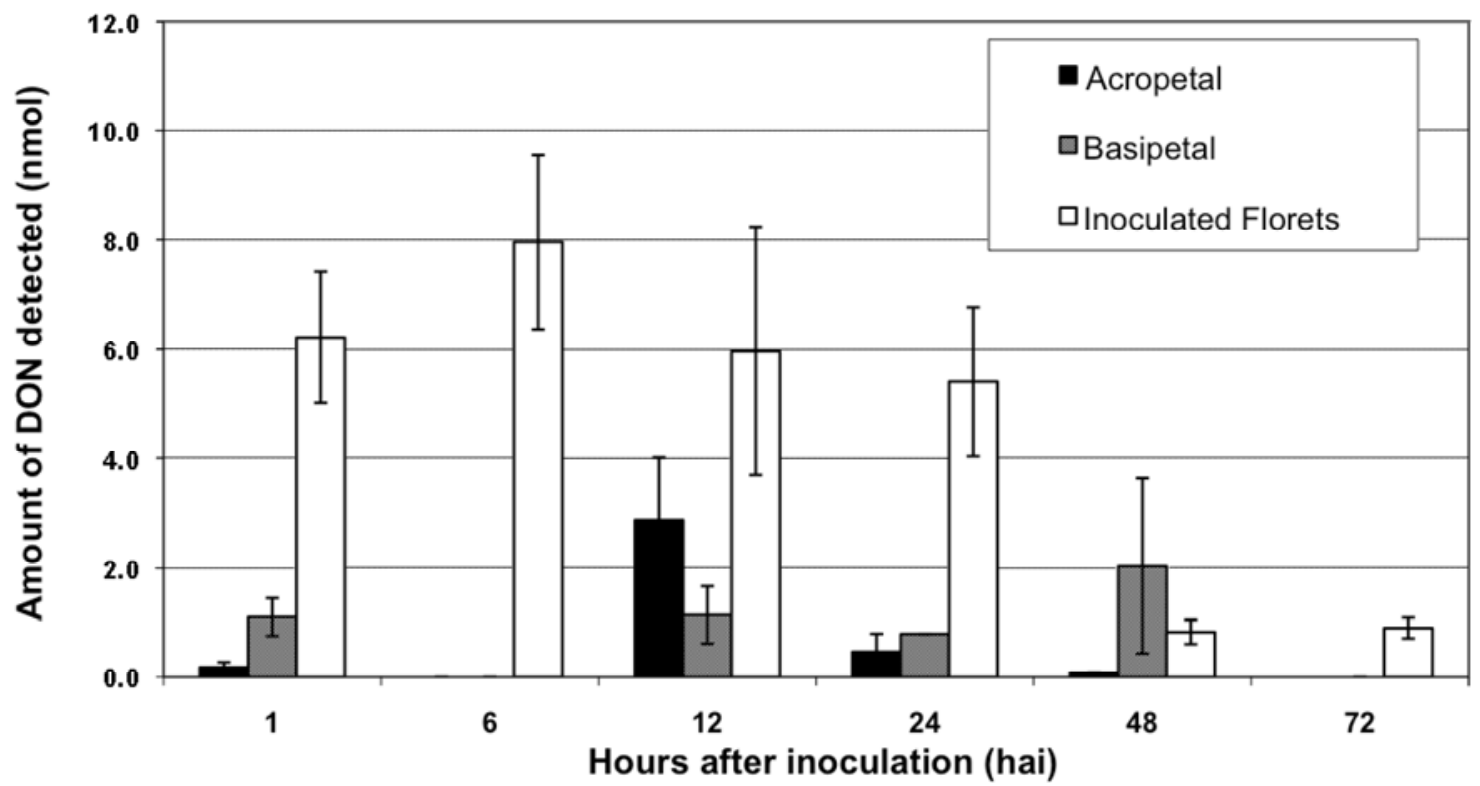

Fig. 1. Deoxynivalenol (DON) concentration ( \pm standard error) in the susceptible barley cv. Morex at $1,6,12,24,48$, and $72 \mathrm{~h}$ after inoculation (hai) in the inoculated florets and in acropetal and basipetal florets at 1, 12, 24, and 48 hai. 
diately above and below the inoculated florets were also analyzed for DON content at 1, 12, 24, and 48 hai. Our results showed that DON moves from the site of inoculation to nearby florets, with levels of DON between 0.08 to $2.88 \mathrm{nmol}$ in acropetal florets and 0.79 to $2.03 \mathrm{nmol}$ detected in basipetal florets. The movement of DON to acropetal versus basipetal florets was not statistically significant.

We also examined the potential of barley to convert DON to D3G. In this experiment, we treated all florets on the barley spikes with $2.0 \mu \mathrm{g}(6.75 \mathrm{nmol})$ of DON per floret and measured DON and D3G concentration. The level of DON in planta decreased $(25.9$ to $9.3 \mu \mathrm{M})$ with time while D3G levels increased ( 0.34 to $2.03 \mu \mathrm{M})$ (Fig. 2). However, only a small proportion (maximum $21.8 \mathrm{~mol} \%$ ) of DON was converted to D3G in this experimental setup.
Barley host response to DON.

The Barley1 GeneChip was used to identify differentiallyaccumulated transcripts in the susceptible barley cv. Morex after DON and water inoculation. We inoculated nine florets in the middle portion of each spike with DON and a water control. Transcript accumulation differences were detected based on $P \leq 0.0001$, false discovery rate (FDR) of $5 \%$, and greater than twofold change in accumulation between the mean values of DON- and water-treated plants. We first assessed the differential transcript accumulation at four timepoints (1, 12, 24, and 48 hai) and detected a total of 685 transcripts exhibiting increased accumulation (all four timepoints combined) and 6 transcripts exhibiting decreased accumulation after DON inoculation compared with water inoculation (Fig. 3). In all, 104, 251, 236, and 94 transcripts showed increased accumulation at

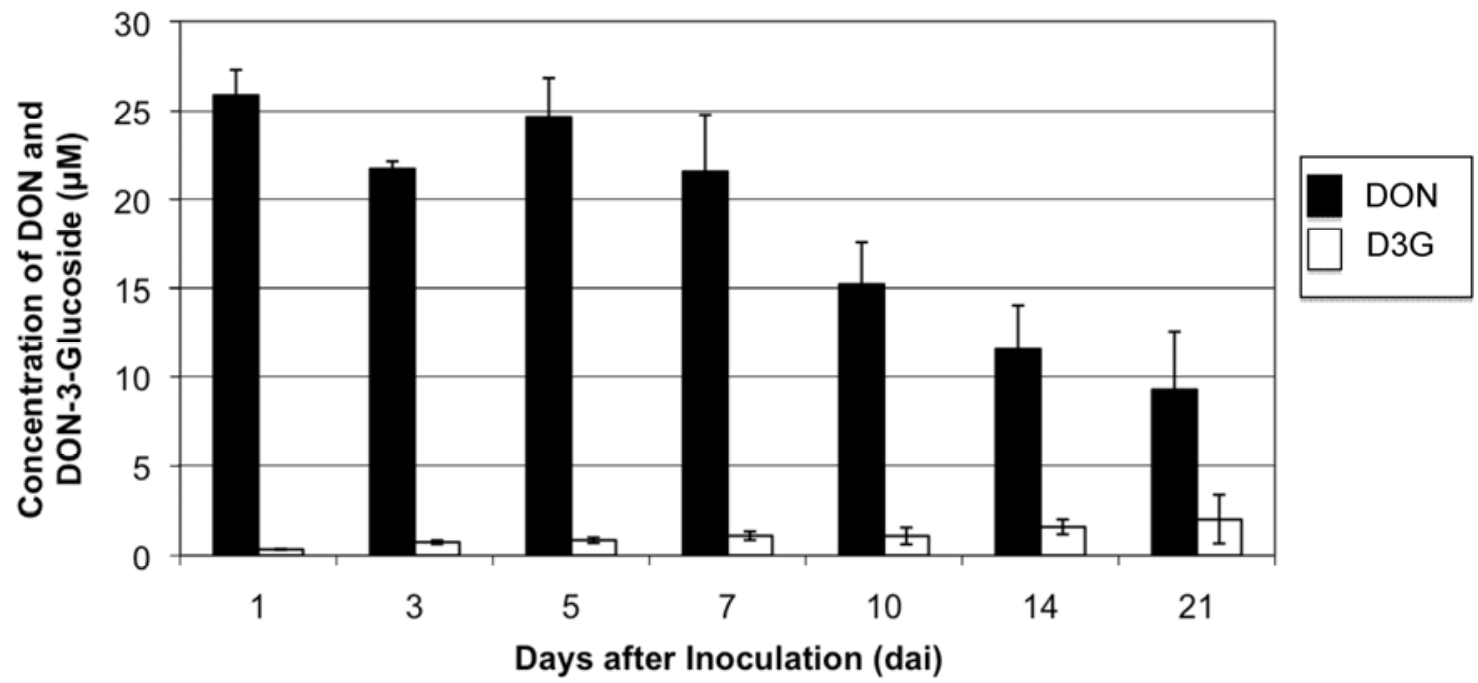

Fig. 2. Deoxynivalenol (DON) and DON-3-O-glucoside (D3G) concentration ( \pm standard error) in the susceptible barley cv. Morex 1 to 21 days after treatment with DON.

\section{A $\quad \mathrm{N}=\sum n=685$}

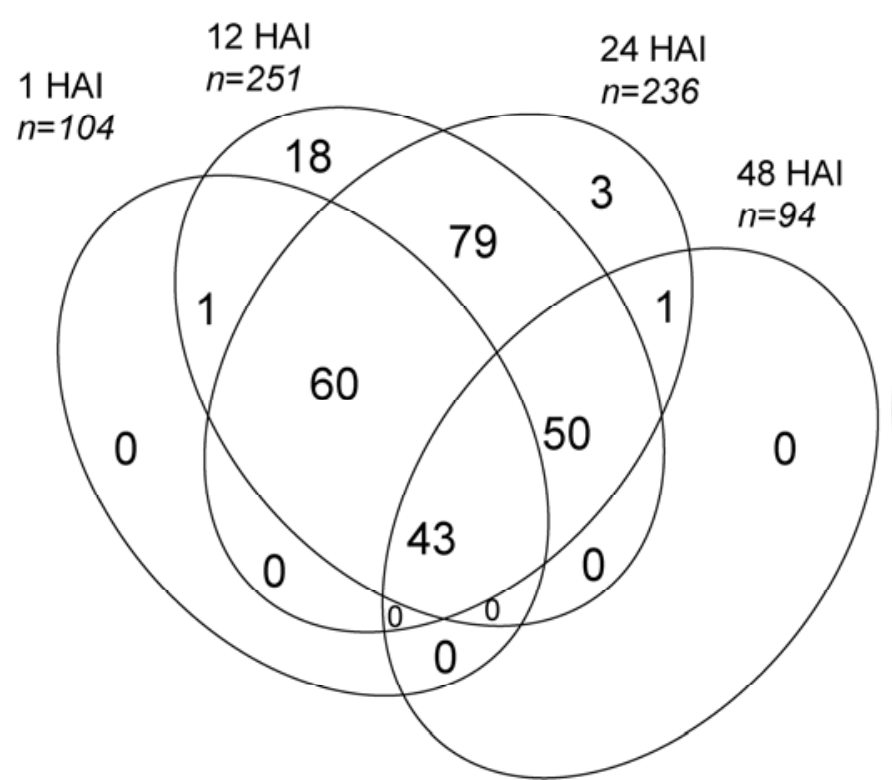

B

$N=\sum n=6$

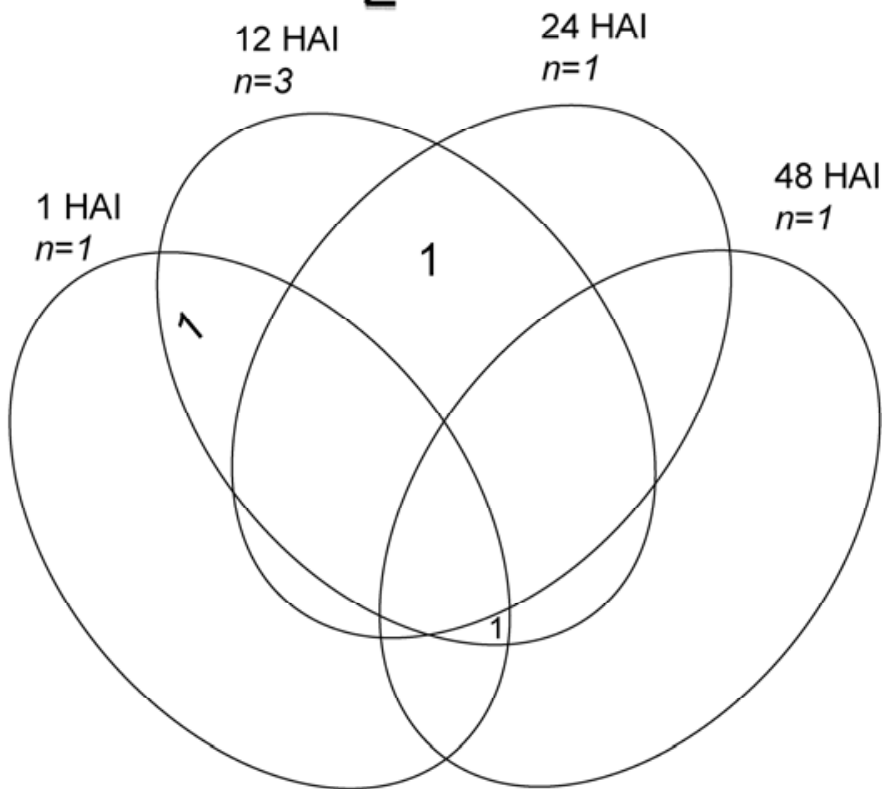

Fig. 3. Pattern of transcript accumulation in deoxynivalenol (DON)-inoculated compared with water mock-inoculated barley at 1 , 12,24 , and 48 h after inoculation (hai). Transcripts were detected based on $P \leq 0.0001$, false discovery rate of $5 \%$, and a twofold difference between the mean values of the DONtreated plants compared with the water-treated plants. A, Venn diagram showing the number of transcripts exhibiting increased accumulation at $1,12,24$, and 48 hai. B, Venn diagram showing the number of transcripts exhibiting decreased accumulation at 1, 12, 24, and 48 hai. 
1, 12, 24, and 48 hai, respectively (Fig. 3A). Considering common as well as exclusive transcripts found across all timepoints, 255 unique transcripts showed increased accumulation and three showed decreased accumulation $(P \leq 0.0001$, FDR $5 \%, \geq$ twofold difference) (Fig. 3; Supplementary Table 1). Forty-three transcripts exhibited increased accumulation in all four timepoints (Fig. 3A). Peak expression values for most transcripts were generally observed at the 12-hai timepoint and leveled off in the subsequent timepoints.

The gene transcripts exhibiting increased accumulation were categorized in the following predicted functional classes: defense, putative trichothecene detoxification, metabolism, regulatory, signal transduction, miscellaneous, hypothetical, and unknown. Of the 255 gene transcripts showing increased accumulation, those encoding regulatory proteins (transcription factors and cys $2 /$ his 2 zinc-fingers) composed the largest annotated functional group at the earlier timepoints (1 and 12 hai), followed closely by those encoding metabolic functions (synthetases and transferases). Transcript accumulation of several functional classes peaked at 12 hai, which included the following: defense (resistance $[R]$ genes and pathogenicity-related), putative trichothecene detoxification (UDP-glucosyltransferases, GSH transferases, ABC transporters, and MATE efflux proteins), and signal transduction (phosphatases, EF-hand proteins and, calmodulin). The highest induction after $12 \mathrm{~h}$ was observed for a putative multidrug resistance protein (182-fold), followed by a cysteine synthase (113-fold) and a putative UDP-glucosyltransferase (82-fold). Three gene transcripts exhibiting reduced accumulation in DON compared with water treatment encoded a boron transporter (Contig19634_at), a glycine-rich cell wall structural protein (Contig11540_s_at), and a $\beta$-glucosidase (Contig8927_at).

\section{Gene transcripts differentially expressed in DON - and $F$. graminearum-treated barley.}

To identify transcript accumulation in response to DON or to pathogen-derived trichothecenes, we conducted a comparative analysis of all transcripts exhibiting increased accumulation with results from two previous studies of the barley- $F$. graminerum interaction (Boddu et al. 2006, 2007). The previous studies examined transcript abundance in barley following $F$. graminearum inoculation compared with a water control (Boddu et al.

Table 1. Transcripts exhibiting increased accumulation detected exclusively by deoxynivalenol (DON) application ${ }^{\mathrm{a}}$

\begin{tabular}{|c|c|c|c|c|c|c|c|c|c|c|c|c|c|c|c|}
\hline \multirow[b]{3}{*}{ Probe set name } & \multirow[b]{3}{*}{ Annotation $^{\mathbf{d}}$} & \multirow[b]{3}{*}{ E value $^{d}$} & \multirow[b]{3}{*}{ Class $^{\mathrm{e}}$} & \multirow{2}{*}{\multicolumn{8}{|c|}{ Transcript accumulation values $^{b}$}} & \multicolumn{4}{|c|}{ Fold change $e^{c}$} \\
\hline & & & & & & & & & & & & \multirow{2}{*}{$\begin{array}{l}\text { D1/ } \\
\text { W1 }\end{array}$} & \multirow{2}{*}{$\begin{array}{l}\text { D12/ } \\
\text { W12 }\end{array}$} & \multirow{2}{*}{$\begin{array}{l}\text { D24/ } \\
\text { W24 }\end{array}$} & \multirow{2}{*}{$\begin{array}{l}\text { D48 } \\
\text { W48 }\end{array}$} \\
\hline & & & & W1 & D1 & W12 & D12 & W24 & D24 & W48 & D48 & & & & \\
\hline Contig10895_at & $\begin{array}{l}\text { Leucine-rich repeat } \\
\text { family protein }\end{array}$ & $2.00 \mathrm{E}-16$ & Defense & 835 & 1,884 & 696.8 & 8,716 & 717.6 & 6,244 & 1,043 & 1,827 & 2.3 & 12.5 & 8.7 & 1.8 \\
\hline Contig7343_s_at & $\begin{array}{l}\text { Serine/threonine protein } \\
\text { kinase }\end{array}$ & $9.00 \mathrm{E}-39$ & Defense & 3,560 & 6,094 & 1,613 & 8,438 & 1,860 & 7,525 & 2,375 & 2,933 & 1.7 & 5.2 & 4 & 1.2 \\
\hline Contig13821_at & $\begin{array}{l}\text { Receptor-like protein } \\
\text { kinase }\end{array}$ & $9.00 \mathrm{E}-85$ & Defense & 266.9 & 360 & 188.6 & 1,504 & 160 & 820.4 & 139.8 & 256.2 & 1.3 & 8 & 5.1 & 1.8 \\
\hline Contig7290_at & $\begin{array}{l}\text { Brassinosteroid } \\
\text { insensitive 1-associated } \\
\text { receptor }\end{array}$ & 0 & Defense & 658.2 & 1,141 & 440.6 & 1,695 & 348.5 & 1,200 & 351.5 & 459.3 & 1.7 & 3.8 & 3.4 & 1.3 \\
\hline Contig11866_at & $\begin{array}{l}\text { Leucine-rich repeat } \\
\text { protein kinase }\end{array}$ & $1.00 \mathrm{E}-151$ & Defense & 253.8 & 435.8 & 197 & 410 & 100.8 & 274.2 & 137.6 & 249.3 & 1.7 & 2.1 & 2.7 & 1.8 \\
\hline Contig6229_s_at & $\begin{array}{l}\text { Putative nematode- } \\
\text { resistance protein }\end{array}$ & $0.00 \mathrm{E}+00$ & Defense & 694 & 1,077 & 711.7 & 1,987 & 408.8 & 1,670 & 369.9 & 620.9 & 1.6 & 2.8 & 4.1 & 1.7 \\
\hline Contig18997_at & $\begin{array}{l}\text { Putative Avr } 9 / \mathrm{Cf}-9 \\
\text { rapidly elicited protein }\end{array}$ & $3.00 \mathrm{E}-79$ & Defense & 153.6 & 922.2 & 78.8 & 1,188 & 75.1 & 981.8 & 90.2 & 101 & 6 & 15.1 & 13.1 & 1.1 \\
\hline Contig13866_at & $\begin{array}{l}\text { Von Willebrand factor } \\
\text { type A (vWA) domain } \\
\text { protein }\end{array}$ & 0 & Metab. & 623.8 & 1,793 & 317.6 & 3,387 & 295.4 & 2,355 & 482.9 & 677.5 & 2.9 & 10.7 & 8 & 1.4 \\
\hline HI05P01u_at & $\begin{array}{l}\text { 2-Oxoglutarate } \\
\text { dehydrogenase E1 } \\
\text { component }\end{array}$ & 0 & Metab. & 350.3 & 621.9 & 323.9 & 979.3 & 292 & 804.8 & 280.7 & 374.8 & 1.8 & 3 & 2.8 & 1.3 \\
\hline Contig3691_at & Aleurone ribonuclease & $1.00 \mathrm{E}-143$ & Metab. & 2,491 & 3,858 & 2,499 & 4,227 & 1,532 & 4,018 & 2,213 & 2,346 & 1.5 & 1.7 & 2.6 & 1.1 \\
\hline EBro04_SQ004_O07a_at & Putative esterase & $4.00 \mathrm{E}-21$ & Metab. & 65.7 & 69 & 64.9 & 1,685 & 61.4 & 986.8 & 67 & 282.8 & 1.1 & 26 & 16.1 & 4.2 \\
\hline Contig19986_at & Lysyl-tRNA synthetase & $6.00 \mathrm{E}-91$ & Metab. & 222 & 325.2 & 210.7 & 6,446 & 226.2 & 3,927 & 224.1 & 657.7 & 1.5 & 30.6 & 17.4 & 2.9 \\
\hline Contig12873_at & $\begin{array}{l}\text { Putative Mal d 1- } \\
\text { associated protein }\end{array}$ & $2.00 \mathrm{E}-72$ & Metab. & 1,071 & 1,480 & 724 & 2,800 & 957.5 & 3,059 & 1,157 & 1,438 & 1.4 & 3.9 & 3.2 & 1.2 \\
\hline Contig17737_at & $\begin{array}{l}\text { Putative linalool } \\
\text { synthase }\end{array}$ & $1.00 \mathrm{E}-105$ & Metab. & 305.4 & 791 & 104.5 & 1,184 & 122.3 & 878.2 & 107.7 & 171.7 & 2.6 & 11.3 & 7.2 & 1.6 \\
\hline Contig1298_at & Enolase & 0 & Metab. & 193.1 & 266.7 & 210.4 & 6,964 & 221.5 & 2,992 & 205.3 & 611.6 & 1.4 & 33.1 & 13.5 & 3 \\
\hline HVSMEa0004N20r2_at & Hydrolase & $1.00 \mathrm{E}-42$ & Metab. & 108.2 & 145 & 82.6 & 394.7 & 81.1 & 234.5 & 93.4 & 106.4 & 1.3 & 4.8 & 2.9 & 1.1 \\
\hline Contig23272_at & Satase isoform & $1.00 \mathrm{E}-66$ & Metab. & 519.1 & 1433 & 590.3 & 4257 & 400.7 & 2722 & 387.1 & 739.6 & 2.8 & 7.2 & 6.8 & 1.9 \\
\hline Contig22479_at & Cytochrome P450 & $4.00 \mathrm{E}-74$ & Metab. & 98.4 & 122.1 & 104.4 & 4906 & 97.8 & 1978 & 97.4 & 417.6 & 1.2 & 47 & 20.2 & 4.3 \\
\hline $\begin{array}{l}\text { Contig11141_at } \\
\text { Contig9662_at }\end{array}$ & $\begin{array}{l}\text { Heat shock protein DnaJ } \\
\text { Monosaccharide }\end{array}$ & $2.00 \mathrm{E}-53$ & Regul. & 317.9 & 398.5 & 247.9 & 767.4 & 288.6 & 594.3 & 319.7 & 324.8 & 1.3 & 3.1 & 2.1 & 1 \\
\hline & transporter 4 & 0 & Regul. & 158.5 & 170.5 & 117.3 & 902.3 & 114.6 & 736.7 & 117.2 & 209.3 & 1.1 & 7.7 & 6.4 & 1.8 \\
\hline Contig20418_at & Dof zinc finger protein 3 & 0 & Regul. & 459.6 & 816.9 & 332.4 & 944.3 & 461.2 & 955.4 & 575.8 & 585.4 & $\begin{array}{l}1.8 \\
(\mathrm{col}\end{array}$ & $\begin{array}{c}2.8 \\
\text { tinued }\end{array}$ & $\begin{array}{c}2.1 \\
\text { on next }\end{array}$ & $\begin{array}{c}1 \\
\text { page })\end{array}$ \\
\hline
\end{tabular}

a Transcripts that were detected in DON-inoculated barley (this study) but not detected in Fusarium graminearum-inoculated barley (Boddu et al. 2006, 2007).

b Mean values of transcript accumulation from DON- and water-treated spikes exhibiting statistically significant differences $(P \leq 0.0001$, false discovery rate $5 \%$, fold-change $\geq$ $2.0)$ were from three replications and were derived from RMA normalized data using the software Genedata Expressionist Pro $($ Genedata, San Francisco $)$. $\mathrm{W}=$ water, $\mathrm{D}=\mathrm{DON}$, and numbers $1,12,24$, and 48 represent hours after inoculation.

${ }^{c}$ Fold-change ratios of $\geq 20$ are indicated in bold.

$\mathrm{d}$ Barley gene annotations were obtained from HarvEST, version 1.64. Annotations were assigned when the BLASTX $e$ value was $<10-10$. N/A $=$ not applicable

${ }^{\mathrm{e}}$ Functional classes: Defense $=$ defense-related proteins; Metab. $=$ proteins with general metabolic functions; Regul. $=$ proteins with regulatory functions; Signal $=$ signal transduction, proteins involved in signaling; Tricho. = proteins related to putative trichothecene detoxification function; Misc. = miscellaneous; and Hypo. $=$ hypothetical proteins or proteins with unknown function. 
2006) and trichothecene-induced transcript abundance in barley following inoculation with a wild-type trichothecene-producing strain and a mutant trichothecene nonproducing strain of the fungus (Boddu et al. 2007). We directly compared probe sets that were identified in the two previous studies with the current study. Of the 255 transcripts that were induced by DON, 93 transcripts were found exclusively in DON-treated barley (Fig. 4A; Table 1). After $12 \mathrm{~h}$, the three most highly upregulated genes of this class were predicted to encode a member of the cytochrome P450 superfamily (47-fold), enolase (33-fold), and lysyl-tRNA synthetase (30-fold). The remaining 162 of the 255 transcripts induced by DON treatment were also found to be induced in $F$. graminearum-infected barley. Boddu and associates (2007) identified 63 transcripts that were classified as induced only by trichothecene-producing $F$. graminearum. Of these 63 trichothecene-specific transcripts, 40 were also found in the current study (Fig. 4B; Table 2). We classified these 40 transcripts as trichothecene-responsive, because they were induced by both DON treatment and pathogen-derived trichothecenes.
The 40 DON-responsive transcripts and pathogen-derived trichothecene-responsive transcripts (Table 2) and the 93 exclusively DON-responsive transcripts (Table 1) provide a combined list of 133 transcripts of particular interest in our study. These transcripts encompassed the following functional classes: defense (kinases and $R$ genes), metabolism (transferases and synthetases), putative trichothecene detoxification (UDP-glucosyltransferases and $\mathrm{ABC}$ transporters), regulatory (ubiquitination, programmed-cell death, and transcription factors), and signal transduction (phosphatases and EF-hand).

\section{Validation of transcript accumulation in response to DON treatment.}

To validate the DON specificity of transcripts detected from the microarray analysis, we conducted quantitative reversetranscriptase polymerase chain reaction (qRT-PCR) on a subset of barley transcripts using a barley line carrying the resistant allele at the chromosome $2 \mathrm{H}$ bin $8 \mathrm{FHB}$ resistance quantitative trait locus (de la Peña et al. 1999, Nduulu et al. 2007). We ex-

Table 1. (continued on next page)

\begin{tabular}{|c|c|c|c|c|c|c|c|c|c|c|c|c|c|c|c|}
\hline \multirow[b]{3}{*}{ Probe set name } & \multirow[b]{3}{*}{ Annotation ${ }^{d}$} & \multirow[b]{3}{*}{ E value ${ }^{d}$} & \multirow[b]{3}{*}{ Class $^{\mathrm{e}}$} & \multirow{2}{*}{\multicolumn{8}{|c|}{ Transcript accumulation values ${ }^{b}$}} & \multicolumn{4}{|c|}{ Fold change ${ }^{c}$} \\
\hline & & & & & & & & & & & & \multirow{2}{*}{$\begin{array}{l}\text { D1/ } \\
\text { W1 }\end{array}$} & \multirow{2}{*}{$\begin{array}{l}\text { D12/ } \\
\text { W12 }\end{array}$} & \multirow{2}{*}{$\begin{array}{l}\text { D24/ } \\
\text { W24 }\end{array}$} & \multirow{2}{*}{$\begin{array}{l}\text { D48/ } \\
\text { W48 }\end{array}$} \\
\hline & & & & W1 & D1 & W12 & D12 & W24 & D24 & W48 & D48 & & & & \\
\hline Contig13835_at & $\begin{array}{l}\text { Putative Myb-like } \\
\text { DNA-binding protein }\end{array}$ & $4.00 \mathrm{E}-21$ & Regul. & 921.7 & 1,637 & 967.3 & 2,017 & 707.4 & 1,946 & 832.8 & 980 & 1.8 & 2.1 & 2.8 & 1.2 \\
\hline Contig24911_at & $\begin{array}{l}\text { AP2 domain containing } \\
\text { protein }\end{array}$ & $4.00 \mathrm{E}-24$ & Regul. & 333.2 & 905.3 & 353.6 & 4,843 & 375.4 & 2,094 & 371.4 & 518.5 & 2.7 & 13.7 & 5.6 & 1.4 \\
\hline Contig20600_at & $\begin{array}{l}\text { Ring-H2 zinc finger } \\
\text { protein }\end{array}$ & $5.00 \mathrm{E}-34$ & Regul. & 247.8 & 437.5 & 230.9 & 1,171 & 211.3 & 677.4 & 210.3 & 328 & 1.8 & 5.1 & 3.2 & 1.6 \\
\hline \multirow[t]{2}{*}{$\begin{array}{l}\text { Contig3323_at } \\
\text { Contig2180_at }\end{array}$} & $\begin{array}{l}\text { BAX inhibitor } \\
\text { F-box domain }\end{array}$ & $1.00 \mathrm{E}-134$ & Regul. & 2,787 & 3,718 & 1,594 & 3,347 & 2,214 & 3,943 & 2,732 & 3,071 & 1.3 & 2.1 & 1.8 & 1.1 \\
\hline & containing protein & $2.00 \mathrm{E}-89$ & Regul. & 612 & 1,030 & 546.1 & 1,447 & 480.5 & 1,055 & 459.9 & 565.6 & 1.7 & 2.6 & 2.2 & 1.2 \\
\hline \multirow{3}{*}{$\begin{array}{l}\text { Contig4387_at } \\
\text { HB25K10r_s_at } \\
\text { HW08K02u_at }\end{array}$} & Putative WRKY & $1.00 \mathrm{E}-178$ & Regul. & 528.4 & 1,814 & 472.3 & 3,858 & 464.5 & 1,881 & 419.2 & 551.9 & 3.4 & 8.2 & 4.1 & 1.3 \\
\hline & Putative WRKY & $1.00 \mathrm{E}-178$ & Regul. & 435.3 & 2,176 & 310.1 & 4,443 & 305.9 & 2,197 & 313.2 & 418.1 & 5 & 14.3 & 7.2 & 1.3 \\
\hline & $\begin{array}{l}\text { Zinc finger transcription } \\
\text { factor }\end{array}$ & $3.00 \mathrm{E}-10$ & Regul. & 190.8 & 350.3 & 137.1 & 433.9 & 146.9 & 261.7 & 146 & 158.9 & 1.8 & 3.2 & 1.8 & 1.1 \\
\hline \multirow[t]{2}{*}{$\begin{array}{l}\text { Contig12925_s_at } \\
\text { Contig16040_at }\end{array}$} & $\begin{array}{l}\text { Scarecrow-like protein } \\
\text { WRKY DNA-binding }\end{array}$ & $1.00 \mathrm{E}-124$ & Regul. & 464 & 948.4 & 434.5 & 1,690 & 414 & 1,189 & 377.5 & 412.4 & 2 & 3.9 & 2.9 & 1.1 \\
\hline & protein & $1.00 \mathrm{E}-133$ & Regul. & 904.3 & 1,078 & 812.1 & 1,749 & 570.1 & 1,573 & 718.1 & 932.6 & 1.2 & 2.2 & 2.8 & 1.3 \\
\hline Contig12925_at & Scarecrow-like protein & $1.00 \mathrm{E}-124$ & Regul. & 65.7 & 170.6 & 65.8 & 339.5 & 53.1 & 236.7 & 47.9 & 61.8 & 2.6 & 5.2 & 4.5 & 1.3 \\
\hline Contig6076_s_at & Ring finger protein & $1.00 \mathrm{E}-114$ & Regul. & 2,368 & 3,821 & 1,296 & 3,083 & 1,781 & 2,959 & 2,283 & 2,393 & 1.6 & 2.4 & 1.7 & 1 \\
\hline Contig7798_at & WRKY & $1.00 \mathrm{E}-136$ & Regul. & 1,902 & 2,954 & 1,562 & 5,035 & 1,196 & 3,705 & 1,198 & 1,449 & 1.6 & 3.2 & 3.1 & 1.2 \\
\hline Contig6727_at & $\begin{array}{l}\text { AP2 transcriptional } \\
\text { activator DRF1 }\end{array}$ & 0 & Regul. & 1,018 & 1,851 & 854.5 & 3,249 & 807.4 & 2,818 & 863.2 & 1,006 & 1.8 & 3.8 & 3.5 & 1.2 \\
\hline Contig9733_s_at & $\begin{array}{l}\text { Zinc finger, } \mathrm{C} 3 \mathrm{HC} 4 \\
\text { family protein }\end{array}$ & $2.00 \mathrm{E}-96$ & Regul. & 497.7 & 719.1 & 536.7 & 1,447 & 517.1 & 953.1 & 561.1 & 624.4 & 1.4 & 2.7 & 1.8 & 1.1 \\
\hline \multirow[t]{2}{*}{ Contig6438_s_at } & $\begin{array}{l}\text { Ring finger protein } \\
\text { DNA-binding protein }\end{array}$ & $1.00 \mathrm{E}-114$ & Regul. & 2368 & 3,821 & 1,296 & 3,083 & 1,781 & 2,959 & 2,283 & 2,393 & 1.6 & 2.4 & 1.7 & 1 \\
\hline & $\begin{array}{l}\text { phosphatase } 2 \mathrm{C} \\
\text { Ethylene-responsive }\end{array}$ & $1.00 \mathrm{E}-168$ & Signal & 1,042 & 1,665 & 519.6 & 3,151 & 458.3 & 2,272 & 711.2 & 1,011 & 1.6 & 6.1 & 5 & 1.4 \\
\hline Contig6636_at & element binding factor & $2.00 \mathrm{E}-92$ & Signal & 833.6 & 1,516 & 649.3 & 1,435 & 569.7 & 1,051 & 620.3 & 678.9 & 1.8 & 2.2 & 1.8 & 1.1 \\
\hline Contig18023_at & Actin binding protein & $4.00 \mathrm{E}-38$ & Signal & 251.2 & 582.9 & 206.6 & 532.6 & 171.7 & 325 & 213.1 & 270.9 & 2.3 & 2.6 & 1.9 & 1.3 \\
\hline Contig6706_at & Sugar transporter family & $1.00 \mathrm{E}-101$ & Signal & 164 & 246.8 & 184.9 & 924.5 & 149.7 & 726.7 & 164.2 & 564.3 & 1.5 & 5 & 4.9 & 3.4 \\
\hline Contig2470_s_at & $\begin{array}{l}\text { Ethylene-responsive } \\
\text { element }\end{array}$ & $2.00 \mathrm{E}-81$ & Signal & 83 & 1,746 & 523.1 & 2,287 & 301.1 & 1,412 & 386.1 & 529.6 & 2.1 & 4.4 & 4.7 & 1.4 \\
\hline Contig11653_at & EF hand family protein & $2.00 \mathrm{E}-65$ & Signal & 166.8 & 265.9 & 154.7 & 407.9 & 140 & 274.2 & 148.1 & 160 & 1.6 & 2.6 & 2 & 1.1 \\
\hline Contig2 & $\begin{array}{l}\text { Protein catalytic/ protein } \\
\text { phosphatase type } 2 \mathrm{C}\end{array}$ & $2.00 \mathrm{E}-43$ & Signal & 433.2 & 587.4 & 321.3 & 1,078 & 292.8 & 749.4 & 282.6 & 364 & 1.4 & 3.4 & 2.6 & 1.3 \\
\hline Contig7514_at & $\begin{array}{l}\text { Signal recognition } \\
\text { particle }\end{array}$ & $2.00 \mathrm{E}-11$ & Signal & 509.9 & 1,538 & 497.4 & 1,331 & 395.1 & 1,172 & 457 & 492.7 & 3 & 2.7 & 3 & 1.1 \\
\hline Contig6131_s_at & $\begin{array}{l}\text { Protein phosphatase } \\
\text { type } 2 \mathrm{C}\end{array}$ & $4.00 \mathrm{E}-75$ & Signal & 1,144 & 1,790 & 1,008 & 2,599 & 1,046 & 1,955 & 921.8 & 1,094 & 1.6 & 2.6 & 1.9 & 1.2 \\
\hline EBro08_SQ004_I20_at & EF hand family protein & $2.00 \mathrm{E}-16$ & Signal & 450.7 & 1,286 & 353.9 & 3,107 & 245.6 & 1,703 & 298.9 & 505.2 & 2.9 & 8.8 & 6.9 & 1.7 \\
\hline Contig19731_at & ATP binding protein & $1.00 \mathrm{E}-11$ & Signal & 51.2 & 60.5 & 49 & 108.8 & 49.9 & 91 & 50.1 & 59 & 1.2 & 2.2 & 1.8 & 1.2 \\
\hline Contig20431_at & $\begin{array}{r}\text { Protein gibberellin } \\
\text { receptor GID1L2 }\end{array}$ & $3.00 \mathrm{E}-54$ & Signal & 504 & 930.2 & 374.3 & 4,317 & 331.3 & 3,957 & 402 & 774.3 & 1.8 & 11.5 & 11.9 & 1.9 \\
\hline Contig19453_at & $\begin{array}{l}\text { Putative zinc } \\
\text { metalloproteinase }\end{array}$ & $3.00 \mathrm{E}-50$ & Signal & 407.6 & 434.5 & 394.8 & 601.9 & 371.5 & 862.7 & 379.2 & 549.1 & 1.1 & 1.5 & 2.3 & 1.4 \\
\hline Contig6557_s_at & Small heat shock protein & $6.00 \mathrm{E}-90$ & Signal & 477.5 & 787.7 & 312.8 & 872.5 & 379.2 & 761.8 & 548.2 & 600.9 & 1.6 & 2.8 & $\begin{array}{l}2 \\
n n e\end{array}$ & $\begin{array}{l}1.1 \\
\text { age })\end{array}$ \\
\hline
\end{tabular}


amined the differential accumulation of transcripts encoding a cytochrome P450 (Contig22479_ at), an NF-X1 transcription factor (Contig7127_at), a gibberellin receptor (Contig20431_at), an F-box domain containing protein (HD08H17r_at), a senescence-associated protein (Contig14507_at), and a UDP-glucosyltransferase (Contig13248_at). Our results show significant differential expression between DON and water-treated samples for 10 of 12 of the timepoint and genotype combinations (Table 3), validating that these genes are induced after DON treatment. Two transcripts were not statistically significant at 1 and 12 hai (Contig14507_at and Contig13248_at, respectively) due to a high level of variation between a single biological replication compared with the two remaining replications.

\section{Formation of DON-GSH conjugates in vitro.}

We detected two classes of genes and obtained preliminary results from functional tests in yeast that indicate that GSH may be important for the host response to DON. We found six GSH-S-transferease (GST) genes that were upregulated in the DON-treated compared with the water-treated plants. GST play a role in detoxification of xenobiotic compounds via conjugation to GSH (Edwards et al. 2000). We also found two cysteine synthases that were upregulated in the DON-treated compared with water-treated plants. Cysteine synthases encode an enzyme in the cysteine biosynthetic pathway, and cysteine is a component of the tripeptide GSH. Thus, increasing the level of cysteine likely increases the level of GSH. In addition, in an independent functional approach, we selected clones from a library of yeast cDNAs (Espinet et al. 1995) overexpressed under the strong GAL1 promoter, which conferred DON resistance in the toxin-sensitive yeast strain YZGA515 (Poppenberger et al. 2003). In this screen, we identified three different classes of inserts (unpublished data). Two independent transformants contained CYS4 (YGR155W), encoding cystathionine $\beta$-syn-

Table 1. (continued on next page)

\begin{tabular}{|c|c|c|c|c|c|c|c|c|c|c|c|c|c|c|c|}
\hline \multirow[b]{3}{*}{ Probe set name } & \multirow[b]{3}{*}{ Annotation $^{d}$} & \multirow[b]{3}{*}{ E value $^{d}$} & \multirow[b]{3}{*}{ Class $^{e}$} & \multirow{2}{*}{\multicolumn{8}{|c|}{ Transcript accumulation values ${ }^{b}$}} & \multicolumn{4}{|c|}{ Fold change $\mathrm{c}$} \\
\hline & & & & & & & & & & & & \multirow{2}{*}{$\begin{array}{l}\text { D1/ } \\
\text { W1 }\end{array}$} & \multirow{2}{*}{$\begin{array}{l}\text { D12/ } \\
\text { W12 }\end{array}$} & \multirow{2}{*}{$\begin{array}{l}\text { D24/ } \\
\text { W24 }\end{array}$} & \multirow{2}{*}{$\begin{array}{l}\text { D48/ } \\
\text { W48 }\end{array}$} \\
\hline & & & & W1 & D1 & W12 & D12 & W24 & D24 & W48 & D48 & & & & \\
\hline Contig7634_at & $\begin{array}{l}\text { Glutathione } \\
\text { S-transferase, } \\
\text { C-terminal domain }\end{array}$ & $5.00 \mathrm{E}-71$ & Tricho. & 608.7 & 705.4 & 624.2 & 1,754 & 558.9 & 1,207 & 587.1 & 769.2 & 1.2 & 2.8 & 2.2 & 1.3 \\
\hline Contig20774_at & $\begin{array}{l}\text { MDR-like ABC } \\
\text { transporter }\end{array}$ & $8.00 \mathrm{E}-46$ & Tricho. & 353.4 & 341.4 & 179.2 & 3,774 & 185.8 & 2,689 & 142.2 & 448.5 & 1 & 21.1 & 14.5 & 3.2 \\
\hline Contig6559_at & $\begin{array}{l}22.3 \mathrm{kDa} \text { heat-shock } \\
\text { protein }\end{array}$ & 0 & Misc. & 1,233 & 1,831 & 929.6 & 1,865 & 1,095 & 1,981 & 1,413 & 1,503 & 1.5 & 2 & 1.8 & 1.1 \\
\hline Contig9576_at & $\begin{array}{l}\text { Metal ion binding } \\
\text { protein }\end{array}$ & $1.00 \mathrm{E}-33$ & Misc. & 735.6 & 1,601 & 593.9 & 2,243 & 567.3 & 1,971 & 527.3 & 665.5 & 2.2 & 3.8 & 3.5 & 1.3 \\
\hline Contig8295_at & $\begin{array}{l}\text { Cotton fiber expressed } \\
\text { protein }\end{array}$ & $6.00 \mathrm{E}-17$ & Misc. & 351.3 & 1,110 & 192.3 & 816.6 & 178.1 & 556.8 & 180.1 & 225.5 & 3.2 & 4.2 & 3.1 & 1.3 \\
\hline HW03O22u_s_at & Secretory protein & $1.00 \mathrm{E}-109$ & Misc. & 298.4 & 362.5 & 308.5 & 2306 & 323.1 & 1199 & 459.7 & 772.9 & 1.2 & 7.5 & 3.7 & 1.7 \\
\hline Contig12124_at & Egg apparatus-1 protein & $3.00 \mathrm{E}-20$ & Misc. & 441.6 & 469 & 472.6 & 990.9 & 453.1 & 966.3 & 429.6 & 734.2 & 1.1 & 2.1 & 2.1 & 1.7 \\
\hline HX14B03r_at & TMV response related & $6.00 \mathrm{E}-18$ & Misc. & 210.6 & 954.8 & 157.5 & 3619 & 104.6 & 2240 & 125.7 & 382.2 & 4.5 & 23 & 21.4 & 3 \\
\hline Contig20919_at & ACR8 protein & $1.00 \mathrm{E}-45$ & Misc. & 454.4 & 751 & 370.3 & 1447 & 337.4 & 1090 & 366.5 & 429.9 & 1.7 & 3.9 & 3.2 & 1.2 \\
\hline Contig11037_at & Metal ion binding & $2.00 \mathrm{E}-51$ & Misc. & 275.9 & 670.4 & 275.3 & 939 & 214.6 & 751.6 & 218.3 & 274.5 & 2.4 & 3.4 & 3.5 & 1.3 \\
\hline Contig14732_at & Nudix hydrolase 4 & $9.00 \mathrm{E}-71$ & Misc. & 365.7 & 699.8 & 248.2 & 945.1 & 222.5 & 1290 & 250.9 & 315.1 & 1.9 & 3.8 & 5.8 & 1.3 \\
\hline Contig3058_at & Metal ion binding & $4.00 \mathrm{E}-85$ & Misc. & 1119 & 2087 & 954.3 & 3933 & 1112 & 2591 & 1228 & 1187 & 1.9 & 4.1 & 2.3 & 1 \\
\hline Contig20575_at & Hairpin-induced protein & $2.00 \mathrm{E}-78$ & Misc. & 214.4 & 272.7 & 222.6 & 638.5 & 201.4 & 444.6 & 200.4 & 253.8 & 1.3 & 2.9 & 2.2 & 1.3 \\
\hline Contig8829_at & Polcalcin Jun o 2 & $2.00 \mathrm{E}-68$ & Misc. & 556.3 & 1000 & 219.8 & 876.4 & 200.8 & 857.3 & 294.8 & 389.7 & 1.8 & 4 & 4.3 & 1.3 \\
\hline Contig7538_at & Copine- 6 & 0 & Misc. & 777.1 & 1537 & 520.1 & 2963 & 361.1 & 1816 & 468.1 & 653.6 & 2 & 5.7 & 5 & 1.4 \\
\hline HS09G19u_s_at & $\begin{array}{l}\text { TIGR01615 family } \\
\text { protein }\end{array}$ & $1.00 \mathrm{E}-115$ & Misc. & 255.6 & 424.5 & 212.6 & 1,918 & 191.9 & 1,112 & 182 & 258.3 & 1.7 & 9 & 5.8 & 1.4 \\
\hline Contig5974_s_at & WIR1A protein & $1.00 \mathrm{E}-23$ & Misc. & 240 & 367.4 & 405.9 & 1,087 & 236.6 & 814.8 & 249.6 & 686.9 & 1.5 & 2.7 & 3.4 & 2.8 \\
\hline Contig15374_at & VQ motif family protein & $1.00 \mathrm{E}-18$ & Misc. & 247 & 608.9 & 244.4 & 2,498 & 210.9 & 1,149 & 204.1 & 347.2 & 2.5 & 10.2 & 5.4 & 1.7 \\
\hline HVSMEn0019K13r2_at & $\begin{array}{l}\text { Armadillo/ } \beta \text {-catenin-like } \\
\text { repeat family protein }\end{array}$ & $5.00 \mathrm{E}-36$ & Misc. & 347.5 & 495.7 & 302.1 & 742.4 & 308.9 & 568.4 & 271.3 & 315.3 & 1.4 & 2.5 & 1.8 & 1.2 \\
\hline rbaal1k11_s_at & Metal ion binding & 0 & Misc. & 1,100 & 1,951 & 795.2 & 3,969 & 940.1 & 2,548 & 1,059 & 1,005 & 1.8 & 5 & 2.7 & 0.9 \\
\hline Contig7378_at & Hypothetical & $2.00 \mathrm{E}-38$ & Нypo. & 1,630 & 2,546 & 1,153 & 3,367 & 982.9 & 2,049 & 1,131 & 1,304 & 1.6 & 2.9 & 2.1 & 1.2 \\
\hline Contig24068_at & Hypothetical & $1.00 \mathrm{E}-11$ & Hypo. & 264.3 & 501.7 & 373.4 & 5,018 & 315.7 & 2,975 & 355.3 & 613.4 & 1.9 & 13.4 & 9.4 & 1.7 \\
\hline Contig10725_at & Hypothetical & $7.00 \mathrm{E}-37$ & Hypo. & 542.9 & 897 & 460.2 & 949.4 & 392.9 & 731.1 & 406.2 & 476.7 & 1.7 & 2.1 & 1.9 & 1.2 \\
\hline EBro01_SQ004_M15_at & Hypothetical & $1.00 \mathrm{E}-13$ & Нypo. & 380.8 & 799.9 & 299.6 & 1,375 & 302.5 & 677 & 273.3 & 345.6 & 2.1 & 4.6 & 2.2 & 1.3 \\
\hline HVSMEf0003G07r2_at & Hypothetical & $2.00 \mathrm{E}-05$ & Hypo. & 834.3 & 1,069 & 599.6 & 1,509 & 672.7 & 1,151 & 722.8 & 762.7 & 1.3 & 2.5 & 1.7 & 1.1 \\
\hline Contig13632_at & Hypothetical & $4.00 \mathrm{E}-53$ & Hypo. & 198 & 236.2 & 184.4 & 1,074 & 178.7 & 440.2 & 174.2 & 322.6 & 1.2 & 5.8 & 2.5 & 1.9 \\
\hline Contig4976_at & Hypothetical & 0 & Hypo. & 1,493 & 4,001 & 976.1 & 6,728 & 499.6 & 5,842 & 540.5 & 1,114 & 2.7 & 6.9 & 11.7 & 2.1 \\
\hline Contig12481_at & Hypothetical & $3.00 \mathrm{E}-10$ & Нypo. & 187.2 & 429.4 & 210.6 & 973.9 & 188 & 529.6 & 188.5 & 206.9 & 2.3 & 4.6 & 2.8 & 1.1 \\
\hline Contig14777_at & Hypothetical & $4.00 \mathrm{E}-63$ & Нyро. & 362 & 576.4 & 282.9 & 1,084 & 263.4 & 590.9 & 275.2 & 311.4 & 1.6 & 3.8 & 2.2 & 1.1 \\
\hline Contig14554_s_at & Hypothetical & $1.00 \mathrm{E}-67$ & Нypo. & 424.5 & 517.8 & 403.3 & 836.7 & 362.1 & 696.9 & 400.6 & 496.3 & 1.2 & 2.1 & 1.9 & 1.2 \\
\hline HVSMEb0007D06r2_x_at & Unknown & $7.00 \mathrm{E}-07$ & Нypo. & 264.1 & 454.4 & 224.4 & 505.9 & 196.6 & 361.5 & 173.5 & 214.2 & 1.7 & 2.3 & 1.8 & 1.2 \\
\hline EBro03_SQ005_G05_at & Unknown & N/A & Hypo. & 190.9 & 1,439 & 144.1 & 1,580 & 125.8 & 561.4 & 143.2 & 163.9 & 7.5 & 11 & 4.5 & 1.1 \\
\hline Contig16356_at & Unknown & N/A & Нypo. & 136.8 & 159.7 & 152.2 & 334.1 & 157.9 & 366.9 & 152.8 & 221.8 & 1.2 & 2.2 & 2.3 & 1.5 \\
\hline Contig20590_at & Unknown & N/A & Hypo. & 218.7 & 381.5 & 216.3 & 471.3 & 218.9 & 535.1 & 178.5 & 210.7 & 1.7 & 2.2 & 2.4 & 1.2 \\
\hline Contig23882_at & Unknown & N/A & Hypo. & 776.9 & 1,366 & 694.3 & 1,707 & 712 & 1,429 & 668.6 & 708.2 & 1.8 & 2.5 & 2 & 1.1 \\
\hline EBpi01_SQ001_G04_at & Unknown & N/A & Нуро. & 285.9 & 563.5 & 251.1 & 1,414 & 248.1 & 1,130 & 312.3 & 400.9 & 2 & 5.6 & 4.6 & 1.3 \\
\hline HB30J05r_at & Unknown & N/A & Нypo. & 504.8 & 690 & 385.4 & 912.9 & 336.2 & 1,209 & 382.7 & 437.3 & 1.4 & 2.4 & 3.6 & 1.1 \\
\hline HVSMEa0007D19r2_at & Unknown & N/A & Нуро. & 62.5 & 81 & 64.9 & 154.1 & 63.7 & 120.3 & 58.3 & 77.4 & 1.3 & 2.4 & 1.9 & 1.3 \\
\hline rbags24b14_at & Unknown & N/A & Нypo. & 26.8 & 123 & 20.9 & 440 & 22.1 & 227.7 & 22.6 & 24.5 & 4.6 & 21.1 & 10.3 & 1.1 \\
\hline Contig11655_at & Unknown & N/A & Нypo. & 349.3 & 601.6 & 288.6 & 2,742 & 241.7 & 1,876 & 257.7 & 405.6 & 1.7 & 9.5 & 7.8 & 1.6 \\
\hline HW03C10u_at & Unknown & N/A & Нypo & 94.5 & 471.2 & 68 & 404 & 68 & 172.4 & 69.4 & 78.8 & 5 & 5.9 & 2.5 & 1.1 \\
\hline Contig14554_at & Unknown & N/A & Нypo. & 280.4 & 334.2 & 258.3 & 509.2 & 240.7 & 445.6 & 266.1 & 312 & 1.2 & 2 & 1.9 & 1.2 \\
\hline
\end{tabular}


thase, which catalyzes the synthesis of cystathionine from serine and homocysteine, the first step in cysteine biosynthesis in yeast. Increased supplementation of liquid medium with either cysteine or GSH increased DON resistance of yeast strain YZGA515 in serial dilution tests (data not shown).

Based on these results, we initiated examination of the potential for the formation of DON-GSH conjugates. We first investigated whether a GSH adduct of DON is formed by living yeast. Evidence for an adduct was found using liquid chromatography-tandem mass spectrometry (LC-MS/MS). However, this adduct was also found in experiments with heat-inactivated yeast, indicating nonenzymatic formation. This could be demonstrated especially in slightly acidic ( $\mathrm{pH}$ 5.0) or alkaline ( $\mathrm{pH}$ 8.0) solutions containing only DON and GSH. MS full scans in atmospheric pressure chemical ionization (APCI) negative mode yielded multiple ions of $\mathrm{m} / \mathrm{z}, 602.3$ at different retention times (Fig. 5). Pure GSH eluted at $5.2 \mathrm{~min}$ and DON at $7.3 \mathrm{~min}$. Multiple peaks of unknown substances have been detected in between those retention times (Fig. 5A). At least three of those compounds are DON-GSH conjugates with a mass of $602.3 \mathrm{amu}$, as shown in the extracted ion chromatograms in Figure 5B (with retention times of approximately 5.7, 6.0, and $6.8 \mathrm{~min}$ ). This mass corresponds to deprotonated ions of true addition products (without the loss of water) of GSH ( $M=307)$ with DON $(M=296)$. MS/MS scans (data not shown), resulted in a neutral loss of the DON moiety and in fragments of GSH, and further structural elucidation or differentiation of the different DON-GSH conjugates by MS/MS scans was not possible due to the similar spectra. Because the products were unstable over time, preparative isolation was not possible. Therefore, nuclear magnetic resonance (NMR) experiments were performed to identify the structures.

For the NMR spectroscopic investigation, DON and GSH were mixed in $\mathrm{D}_{2} \mathrm{O}$ and ${ }^{1} \mathrm{H}$ spectra were recorded after $3 \mathrm{~h}, 1$ day, and 3 days (Fig. 6). After 3 h, a substantial amount of
DON (Fig. 6B) was still present (the peaks at 6.4, 3.75, 2.1, and $1.7 \mathrm{ppm}$ can easily be followed even in the mixture), and there was one major reaction product (Fig. 6, peaks at 0.9, $0.92,3.3,4.0$, and 4.48). At the 1-day reaction time, DON was almost completely gone and a complex mixture of reaction products appeared. After two more days, some more minor changes in the product composition could be observed but, thereafter, the reactions seemed to have ceased. The final mixture at that stage consisted of at least five compounds in differing amounts. Clearly, there was a continuing decrease in the amount of unreacted GSH present, which can be followed using the signals at $4.17,2.79$, and $2.68 \mathrm{ppm}$ representing the $\alpha$ and $\beta$ protons of the cysteine moiety.

Unfortunately, it was not possible to completely elucidate the structures of the individual reaction products. On the one hand, the time at which one reaction product dominates was too short for full characterization (a few hours); on the other hand, the final reaction mixture was too complex for individual compounds to be evaluated even by a full set of two-dimensional spectra due to heavy signal overlap. However, some information could be revealed from the ${ }^{1} \mathrm{H}$ spectra as well as from the two-dimensional spectra recorded between day one and day three of the reaction. DON and each of its derivatives have two methyl groups with characteristic shifts in both ${ }^{1} \mathrm{H}$ (1.8 to $0.8 \mathrm{ppm})$ and ${ }^{13} \mathrm{C}$ spectra $(20$ to $10 \mathrm{ppm})$; thus, the number of reaction products could be estimated as half the number of peaks in the respective areas. Because all of the proton resonances of the major reaction products were found below $1.2 \mathrm{ppm}$, it is clear that the respective methyl groups cannot be attached to an olefinic moiety, in contrast to C-16 in the parent DON structure. Furthermore, all of these have to be attached to a quaternary carbon atom due to their lack of $\mathrm{H}-\mathrm{H}$ couplings (as seen in the COSY spectrum). These conditions can only be met if a tautomeric rearrangement takes place before or after the nucleophilic addition of the $\mathrm{SH}$ group to the

\section{A}

Wildtype F. graminearuminduced transcripts (467)

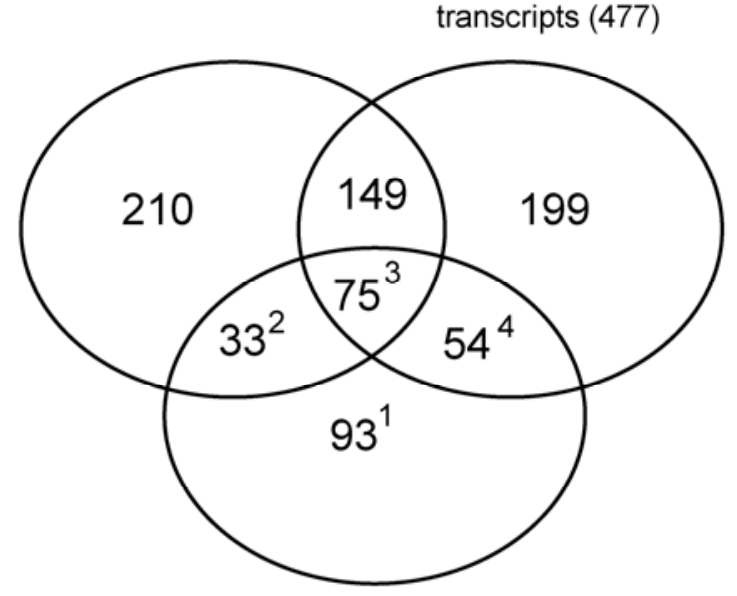

Wildtype $F$. graminearum-, and Tri5 mutant Fusarium- induced transcripts (477)

\section{DON-induced transcripts \\ (DON inoculation) (255)}

B

$\begin{array}{ll}\text { Transcripts induced in both } & \text { Trichothecene-specific } \\ \text { Boddu et. al. 2007 study and } & \text { transcripts from Boddu et. } \\ \text { DON study (129) } & \text { al. } 2007(63)\end{array}$

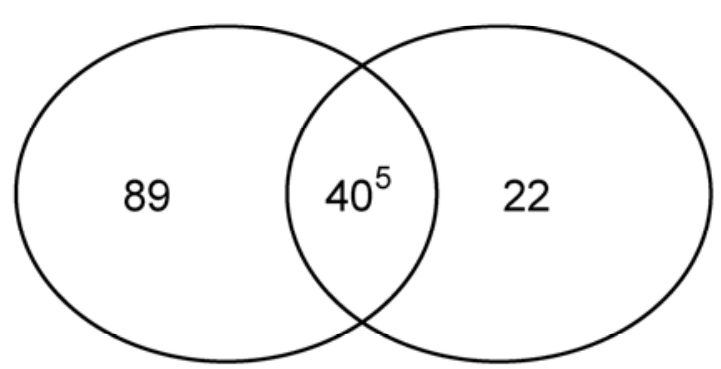

Fig. 4. Comparison of the number of differentially accumulating transcripts from deoxynivalenol (DON) application to two previous Fusarium graminearum-induced transcript accumulation studies. A, In all, 467 transcripts were detected in wild-type F. graminearum-infected barley (Boddu et al. 2006) and 477 transcripts were detected in wild-type F. graminearum-infected or Tri5 mutant $F$. graminearum-infected barley (Boddu et al. 2007). 1, Ninety-three genes induced by DON application. 2, Thirty-three genes induced by wild-type $F$. graminearum inoculation and DON application (Supplementary Table 2). 3, Seventy-five transcripts shown to be induced in all three studies (Supplementary Table 3). 4, Fifty-four transcripts induced by wild-type F. graminearum or Tri5 mutant F. graminearum and DON application (Supplementary Table 4). B, In total, 129 transcripts identified by Boddu and associates (2007) and in the current study were compared with the 63 trichothecene-specific transcripts identified by Boddu and associates (2007). 5, A subset of 40 transcripts was detected in both studies and classified as trichothecene specific. 
Table 2. Barley transcripts exhibiting increased accumulation to deoxynivalenol (DON) and in planta Fusarium graminearum-derived trichothecenes ${ }^{\mathrm{a}}$

\begin{tabular}{|c|c|c|c|c|c|c|c|c|c|c|c|c|c|c|c|}
\hline \multirow[b]{3}{*}{ Probe set name } & \multirow[b]{3}{*}{ Annotation ${ }^{d}$} & \multirow[b]{3}{*}{ E value $^{\text {d }}$} & \multirow[b]{3}{*}{ Class $^{\mathrm{e}}$} & \multirow{2}{*}{\multicolumn{8}{|c|}{ Transcript accumulation values $^{b}$}} & \multicolumn{4}{|c|}{ Fold change $^{c}$} \\
\hline & & & & & & & & & & & & \multirow{2}{*}{$\begin{array}{l}\text { D1/ } \\
\text { W1 }\end{array}$} & \multirow{2}{*}{$\begin{array}{c}\text { D12 } \\
\text { /W12 }\end{array}$} & \multirow{2}{*}{$\begin{array}{c}\text { D24 } \\
\text { /W24 }\end{array}$} & \multirow{2}{*}{$\begin{array}{l}\text { D48/ } \\
\text { W48 }\end{array}$} \\
\hline & & & & W1 & D1 & W12 & D12 & W24 & D24 & W48 & D48 & & & & \\
\hline VSMEk0004K15r2_at & $\begin{array}{l}\text { Leucine-rich repeat } \\
\text { family }\end{array}$ & $3.00 \mathrm{E}-48$ & Defense & 587.6 & 994.2 & 515.4 & 4758 & 509.9 & $3,316.3$ & 528.8 & 898.9 & 1.7 & 9.2 & 6.5 & 1.7 \\
\hline Contig19921_at & $\begin{array}{l}\text { Putative immediate- } \\
\text { early fungal elicitor } \\
\text { protein }\end{array}$ & $2.00 \mathrm{E}-35$ & Defense & 420.8 & 785.5 & 127.3 & 2,013 & 75.7 & $1,206.8$ & 76.8 & 312.6 & 1.9 & 15.8 & 15.9 & 4.1 \\
\hline Contig4640_s_at & $\begin{array}{l}\text { Metal ion binding } \\
\text { protein }\end{array}$ & $5.00 \mathrm{E}-37$ & Metab. & 501.8 & 923.9 & 358.9 & $1,213.1$ & 295.6 & 677.6 & 360.7 & 437.2 & 1.8 & 3.4 & 2.3 & 1.2 \\
\hline Contig9265_at & $\begin{array}{l}\text { Serine/threonine } \\
\text { phosphatase type } 2 \mathrm{c}\end{array}$ & $1.00 \mathrm{E}-124$ & Metab. & 396.6 & $1,874.2$ & 341.2 & $1,970.1$ & 193.6 & 757.6 & 182.8 & 283.9 & 4.7 & 5.8 & 3.9 & 1.6 \\
\hline HV12E23u_at & Cysteine synthase & $1.00 \mathrm{E}-58$ & Metab. & 58.9 & 138.3 & 58.6 & 6,650 & 66.7 & 4,920 & 60.7 & 542.7 & 2.3 & 113.5 & 73.7 & 8.9 \\
\hline Contig12075_at & $\begin{array}{l}\text { Protein flavonol 4- } \\
\text { sulfotransferase }\end{array}$ & $1.00 \mathrm{E}-132$ & Metab. & 948.2 & $3,477.8$ & 667.3 & $9,718.8$ & 526.9 & $7,774.5$ & 465.7 & $1,597.8$ & 3.7 & 14.6 & 14.8 & 3.4 \\
\hline Contig20974_at & $\begin{array}{l}\text { Cytochrome } \mathrm{P} 450 \\
\text { related cluster }\end{array}$ & $1.00 \mathrm{E}-98$ & Metab. & 615.1 & $1,471.4$ & 371.1 & $13,604.9$ & 300.3 & $9,771.6$ & 333.8 & $1,531.1$ & 2.4 & 36.7 & 32.5 & 4.6 \\
\hline Contig11818_at & $\begin{array}{l}\text { Cytochrome } \mathrm{P} 450 \\
\text { related cluster }\end{array}$ & $1.00 \mathrm{E}-147$ & Metab. & 594.2 & $2,244.5$ & 674 & $8,240.9$ & 342.2 & $7,223.1$ & 289.7 & $1,380.1$ & 3.8 & 12.2 & 21.1 & 4.8 \\
\hline EBro08_SQ004_B22_at & $\begin{array}{l}\text { Cytochrome } \mathrm{P} 450 \\
\text { related cluster }\end{array}$ & $3.00 \mathrm{E}-55$ & Metab. & 90.2 & 100.9 & 97.9 & $6,387.1$ & 103 & $5,913.2$ & 95.9 & 791.6 & 1.1 & 65.3 & 57.4 & 8.3 \\
\hline Contig18990_at & $\begin{array}{l}\text { Cytochrome } \mathrm{P} 450 \\
\text { related cluster }\end{array}$ & $6.00 \mathrm{E}-86$ & Metab. & 191.4 & 217.2 & 162.5 & $3,205.6$ & 145.9 & $1,934.2$ & 147.5 & 427.7 & 1.1 & 19.7 & 13.3 & 2.9 \\
\hline Contig3047_s_at & Cytochrome P450 & $1.00 \mathrm{E}-142$ & Metab. & 391.2 & $1,036.7$ & 219.1 & $4,318.9$ & 178.9 & $3,832.4$ & 152.2 & 541 & 2.6 & 19.7 & 21.4 & 3.6 \\
\hline Contig20981_at & Cys2/His2 zinc-finger & $9.00 \mathrm{E}-42$ & Regul. & 178.7 & $1,466.7$ & 48.1 & 2,963 & 51.9 & $1,682.5$ & 54.6 & 297.4 & 8.2 & 61.7 & 32.4 & 5.4 \\
\hline Contig21462_at & Cys2/His2 zinc-finger & $6.00 \mathrm{E}-12$ & Regul. & 249.9 & 679.6 & 137.6 & $1,857.4$ & 141.5 & 993.2 & 146.1 & 229.9 & 2.7 & 13.5 & 7 & 1.6 \\
\hline Contig12590_at & $\begin{array}{l}\text { Putative regulator of } \\
\text { gene silencing }\end{array}$ & $8.00 \mathrm{E}-48$ & Regul. & 620.1 & $2,040.1$ & 279.2 & $4,836.1$ & 313.6 & 3,877 & 289.8 & 951.9 & 3.3 & 17.3 & 12.4 & 3.3 \\
\hline EBro02_SQ005_B08_at & Cys2/His2 zinc-finger & $4.00 \mathrm{E}-12$ & Regul. & 405.6 & $1,131.5$ & 213 & 743.2 & 46.9 & 114.9 & 55 & 62.3 & 2.8 & 3.5 & 2.5 & 1.1 \\
\hline Contig14507_at & $\begin{array}{l}\text { SAG21 (senescence- } \\
\text { associated gene 21) }\end{array}$ & $3.00 \mathrm{E}-10$ & Regul. & 265.7 & 438.1 & 142.8 & $9,500.2$ & 144.1 & 4,679 & 155.7 & 745.6 & 1.6 & 66.5 & 32.5 & 4.8 \\
\hline Contig19256_at & $\begin{array}{l}\text { Mitogen-activated } \\
\text { protein kinase }\end{array}$ & $1.00 \mathrm{E}-101$ & Regul. & 185.4 & 736.3 & 118 & 2,408 & 131.5 & $1,873.3$ & 142.8 & 363.2 & 4 & 20.4 & 14.2 & 2.5 \\
\hline Contig23834_at & $\begin{array}{l}\text { Mitogen-activated } \\
\text { protein kinase }\end{array}$ & 41 & Regul. & 112.6 & 276.1 & 100.2 & 2,0 & 93.7 & 1,3 & 92.9 & 24 & 2.5 & 20.3 & 14.7 & 2.7 \\
\hline Contig8220_at & F-box domain & $4.00 \mathrm{E}-55$ & Regul. & 492.3 & $1,124.8$ & 278.2 & $4,824.5$ & 275.4 & $2,991.8$ & 279.2 & 519.4 & 2.3 & 17.3 & 10.9 & 1.9 \\
\hline HD08H17r_at & F-box domain & $2.00 \mathrm{E}-15$ & Regul. & 246.1 & 496.5 & 261.2 & 5,856 & 248.3 & $2,732.2$ & 251 & 519.1 & 2 & 22.4 & 11 & 2.1 \\
\hline Contig7127_at & $\begin{array}{l}\text { Transcriptional } \\
\text { repressor NF-X1 }\end{array}$ & $0.00 \mathrm{E}+00$ & Regul. & 383.8 & 915.9 & 360.2 & $3,568.9$ & 336.5 & $2,247.2$ & 325.9 & 592.4 & 2.4 & 9.9 & 6.7 & 1.8 \\
\hline Contig7132_s_at & $\begin{array}{l}\text { Transcriptional } \\
\text { repressor NF-X1 }\end{array}$ & $0.00 \mathrm{E}+00$ & Regul. & 439.4 & $1,102.8$ & 386.6 & $4,438.2$ & 349.4 & $3,078.5$ & 337.8 & 733 & 2.5 & 11.5 & 8.8 & 2.2 \\
\hline HVSMEm0012J18r2_at & $\begin{array}{l}\text { Putative } O \text { - } \\
\text { glucosyltransferase }\end{array}$ & $4.00 \mathrm{E}-62$ & Tricho. & 187.9 & 414.3 & 172.2 & $2,847.9$ & 150.1 & 1,504 & 143.5 & 249.9 & 2.2 & 16.5 & 10 & 1.7 \\
\hline Contig13248_at & $\begin{array}{l}\text { UDP- } \\
\text { glucosyltransferase }\end{array}$ & $3.00 \mathrm{E}-90$ & Tricho. & 238.1 & $1,114.9$ & 126.3 & 10,447 & 94.3 & 9,046 & 71.7 & 1,7 & 4.7 & 82.7 & 95.9 & 25.1 \\
\hline Contig9422_at & Multic & $1.00 \mathrm{E}-141$ & Tricho. & 508.7 & $1,549.6$ & 375.9 & 7973.9 & 250.1 & $6,629.5$ & 238 & $1,015.3$ & 3 & 21.2 & 26.5 & 4.3 \\
\hline Contig21298_at & Multidrug resistance & $2.00 \mathrm{E}-89$ & Tricho. & 68.3 & 313.4 & 60.2 & $10,964.3$ & 52.8 & $7,255.6$ & 48.3 & 660.9 & 4.6 & 182.1 & 137.4 & 13.7 \\
\hline Contig13952_at & TMV-response related & $6.00 \mathrm{E}-52$ & Misc. & 103.2 & 398.2 & 114.5 & $1,407.2$ & 81.5 & $1,026.1$ & 79 & 182.5 & 3.9 & 12.3 & 12.6 & 2.3 \\
\hline Contig15142_at & $\begin{array}{l}\text { Mitochondrial } \\
\text { chaperone BCS1 }\end{array}$ & $1.00 \mathrm{E}-164$ & Misc. & 305.6 & 459.4 & 196 & $4,241.7$ & 180.6 & $2,891.9$ & 201.7 & 426.5 & 1.5 & 21.6 & 16 & 2.1 \\
\hline Contig15757_at & $\begin{array}{l}\text { Mitochondrial } \\
\text { chaperone BCS1 }\end{array}$ & $1.00 \mathrm{E}-170$ & Misc. & 463.6 & & 383 & & 311.3 & 73 & 386.3 & & 1.4 & 2.7 & 2.4 & 1.3 \\
\hline Contig12445_at & Protein ATP8 & $1.00 \mathrm{E}-111$ & Misc. & 1,069 & $2,285.8$ & 556.8 & $11,219.9$ & 599.6 & $8,768.3$ & 634 & $1,765.2$ & 2.1 & 20.2 & 14.6 & 2.8 \\
\hline Contig15650_at & Hypothetical & $7.00 \mathrm{E}-38$ & Нypo. & 325.9 & $1,057.7$ & 228.3 & $3,841.1$ & 238 & $1,897.8$ & 265.4 & 406.5 & 3.2 & 16.8 & 8 & 1.5 \\
\hline Contig19553_at & Hypothetical & $6.00 \mathrm{E}-54$ & Нypo. & 342.1 & $1,219.4$ & 245.6 & $6,270.4$ & 229 & $5,439.3$ & 199 & 944.9 & 3.6 & 25.5 & 23.8 & 4.7 \\
\hline Contig15187_at & Hypothetical & $2.00 \mathrm{E}-32$ & Нypo. & 488.5 & $1,632.1$ & 364.9 & $2,745.4$ & 396.4 & $2,329.3$ & 356 & 514.2 & 3.3 & 7.5 & 5.9 & 1.4 \\
\hline Contig17926_at & Hypothetical & $1.00 \mathrm{E}-10$ & Нypo. & 779.5 & 983.3 & 430.7 & 6,835 & 611.6 & $7,238.8$ & 615.7 & $1,185.3$ & 1.3 & 15.9 & 11.8 & 1.9 \\
\hline Contig12633_at & Unknown & $0.00 \mathrm{E}+00$ & Нypo. & 909.1 & 1621.3 & 457.5 & $10,424.1$ & 332.4 & $8,198.7$ & 430 & $1,948.3$ & 1.8 & 22.8 & 24.7 & 4.5 \\
\hline Contig14114_at & Unknown & $0.00 \mathrm{E}+00$ & Нypo. & 59.8 & 132.4 & 40 & 443.8 & 38.2 & 322.9 & 44.1 & 60.9 & 2.2 & 11.1 & 8.5 & 1.4 \\
\hline Contig20755_at & Unknown & $0.00 \mathrm{E}+00$ & Нypo. & 181.4 & 919.4 & 119.4 & $7,584.9$ & 132.3 & $6,813.5$ & 127.3 & 908.7 & 5.1 & 63.5 & 51.5 & 7.1 \\
\hline EBro02_SQ006_C05_at & Unknown & $0.00 \mathrm{E}+00$ & Нypo. & 66.1 & 288 & 40.8 & $2,048.1$ & 34.9 & $1,254.5$ & 37.6 & 173.3 & 4.4 & 50.2 & 36 & 4.6 \\
\hline HO10M16S_at & Unknown & $0.00 \mathrm{E}+00$ & Нypo. & 555 & $4,052.2$ & 40.8 & $2,048.1$ & 248.9 & 11,142 & 222.6 & $2,016.8$ & 7.3 & 50.2 & 44.8 & 9.1 \\
\hline HVSMEa0006G22r2_at & Unknown & $0.00 \mathrm{E}+00$ & Нypo. & 253.1 & 357 & 290.7 & $1,798.5$ & 278.4 & $2,635.9$ & 286 & 644.9 & 1.4 & 6.2 & 9.5 & 2.3 \\
\hline
\end{tabular}

${ }^{a}$ Transcripts identified as trichothecene specific during in planta trichothecene accumulation by Boddu and associates (2007) and also detected as DON responsive in this study.

${ }^{\mathrm{b}}$ Mean values of transcript accumulation from DON- and water-treated spikes exhibiting statistically significant differences (analysis of variance, $P \leq$ 0.0001; false discovery rate 5\%; fold-change $\geq 2.0$ ) were from three replications and were derived from RMA-normalized data using the software Genedata Expressionist Pro (Genedata, San Francisco).

${ }^{c}$ Fold-change ratios $\geq 20$ are indicated in bold and fold-change ratios $\geq 50$ are indicated in bold italics.

d Barley gene annotations were obtained from HarvEST version 1.64. Annotations were assigned when the BLASTX e value was $<10-10$. W and D $=$ water, DON and numbers $1,12,24$, and 48 represent hours after inoculation.

${ }^{\mathrm{e}}$ Functional classes: Defense $=$ defense-related proteins; Metab. = proteins with general metabolic functions; Regul. = proteins with regulatory functions; Signal = signal transduction, proteins involved in signaling; Tricho. $=$ proteins related to putative trichothecene detoxification function; Misc. $=$ miscellaneous; and Hypo. = hypothetical proteins or proteins with unknown function. 
DON molecule, a process which is surely facilitated by the alkaline aqueous medium. However, there is evidence that the two expected compounds (addition at the keto group or addition at the double bond) may also be present within the minor reaction products: a $\mathrm{CH}_{3}$ group coupled to a neighboring $\mathrm{CH}$ and showing a long range correlation to a ketone carbon atom is the result of a Michael-type addition of the GSH SH group to the enone moiety of DON; and a methyl signal similar to DON coupled to a less deshielded olefinic proton (5.49 compared with $6.40 \mathrm{ppm}$ of its DON analog), together with a ketal-type carbon signal (107 ppm), indicate the formation of a mono-thio ketal by the addition of the thiol to the carbonyl group itself.

\section{DISCUSSION}

Our aims were to characterize the fate and movement of DON in barley and to identify barley genes that respond specifically to DON. Thus, identifying genes that respond to DON treatment may uncover genes that reduce the impact of FHB. Two previous studies examined the barley transcriptome during $F$. graminearum infection (Boddu et al. 2006, 2007) but these studies did not examine the barley transcriptome after
DON treatment. Studies examining the host response to direct trichothecene treatment in Arabidopsis suggest there are genetic mechanisms specifically targeting DON (Poppenberger et al. 2003, Asano et. al. 2007; Masuda et al. 2007). RNA profiling of DON-treated wheat revealed that lines containing the Fhbl resistance allele showed increased accumulation of transcripts encoding proteins involved in early host defense to DON (Walter et. al. 2008). In contrast, Desmond and associates (2008) found evidence of apoptosis in DON-treated wheat plants, such as DNA laddering. Our study provides the opportunity to examine the fate of DON, describe the patterns of barley transcript accumulation in response to DON application, examine the fate of DON in relation to the transcript accumulation patterns, identify key genes potentially involved in host defense to DON, and examine the in vitro formation of DON-GSH conjugates.

\section{The fate of DON in barley.}

We observed that, as the concentration of DON decreased in the inoculated florets, DON increased in the adjacent acropetal and basipetal noninoculated florets (Fig. 1). The subcellular movement of DON has been previously described in wheat

Table 3. Validation of deoxynivalenol (DON) induced transcripts

\begin{tabular}{|c|c|c|c|}
\hline \multirow[b]{2}{*}{ Probe set } & \multirow[b]{2}{*}{ Annotation } & \multicolumn{2}{|c|}{ Range of fold change ratios ${ }^{a}$} \\
\hline & & $1 \mathrm{~h}$ & $12 \mathrm{~h}$ \\
\hline Contig7127_at & NF-X1 & 17.43 to $42.32 * * *$ & 4.891 to $144.3^{*}$ \\
\hline Contig22479_at & Cytochrome P450 & 10.01 to $99.27 *$ & 891.4 to $2568 * * *$ \\
\hline Contig13248_at & UDP-glucosyltransferase & 31.78 to $1555^{*}$ & 0.824 to 527.6 \\
\hline HD08H17r_at & F-box & 15.63 to $266.3^{*}$ & 150.8 to $947.7 * * *$ \\
\hline Contig20431_at & Gibberellin receptor & 8.674 to $169.3^{*}$ & 2.848 to $27.67 *$ \\
\hline Contig14507_at & Senescence-associated & 0.920 to 6.498 & 1,915 to $13,216^{* * * *}$ \\
\hline
\end{tabular}

${ }^{a}$ Range of fold-change ratios of DON- to water-treated samples in three biological replications of a genotype carrying the Chevron resistance allele at the chromosome $2 \mathrm{H}$ bin 8 quantitative trait locus are shown. Fold-changes were calculated using a primer efficiency of 2 , such that fold-change $=2^{\wedge}\left(\Delta \mathrm{Ct} t_{\text {target }}-\right.$ $\left.\Delta \mathrm{Ct}_{\text {reference }}\right)=2^{\wedge}\left[\left(\mathrm{Ct}_{\text {target, H2O }}-\mathrm{Ct}_{\text {target, DON }}\right)-\left(\mathrm{Ct}_{\text {reference, H2O }}-\mathrm{Ct}_{\text {reference, DON }}\right)\right]$, where $\mathrm{Ct}=$ cycle threshold. A barley $\alpha$-tubulin was used as the reference gene; 1 and $12 \mathrm{~h}$ indicates 1 and $12 \mathrm{~h}$ after inoculation, respectively. The level of significance of the mean $\Delta \Delta \mathrm{Ct}$ of a target gene in DON- compared with water-treated samples are indicated as $* * *, * *$, and $*=P<0.01,0.02$, and 0.05 , respectively.

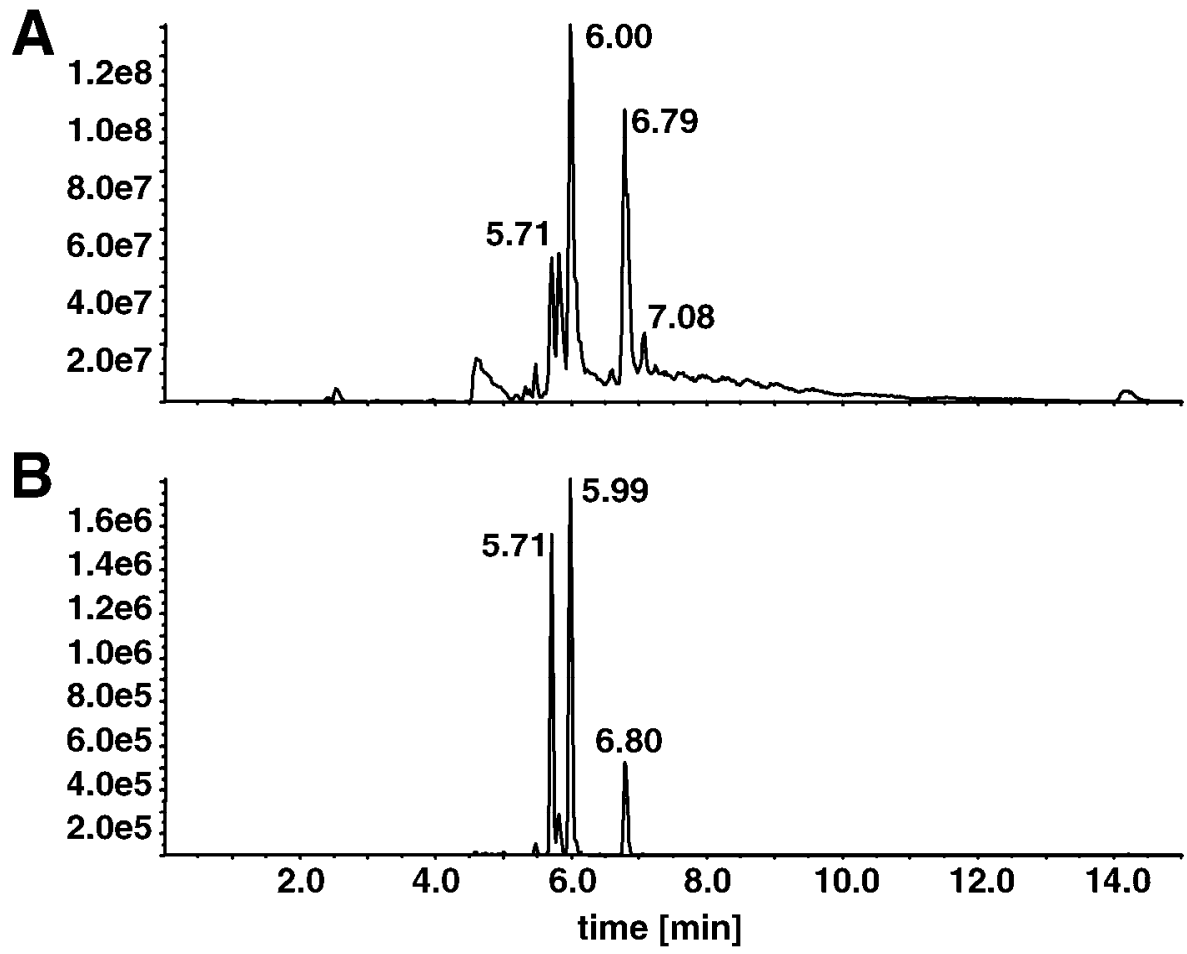

Fig. 5. Liquid chromatography atmospheric pressure chemical ionization EMS chromatograms of deoxynivalenol (DON) plus glutathione (GSH). A, Total ion chromatogram (250 to 1,000 amu). B, Extracted ion chromatogram (602 to $603 \mathrm{amu}$ ), corresponding to the [M-H] ${ }^{-}$ion of DON-GSH. 
during F. culmorum infection (Kang and Buchenauer 1999). They detected DON in phloem and xylem tissues above the site of inoculation as well as in phloem tissues below the site of infection. Their results suggest that DON can move along with water and photosynthates within the plant. Savard and associates (2000) measured DON accumulation in F. graminearum-treated wheat tissue from 4 to 25 days after infection. These authors found that DON moved primarily downward through the spike from the point of inoculation. In our study, DON was detected in acropetal and basipetal florets but statistical significance was not found showing a preferential movement in either direction. In addition, our measurements were conducted after DON inoculation from 1 to 72 hai for parallel assessment with the microarray data as opposed to over a period of weeks. However, the results indicate that movement of DON to nearby florets both below and above the site of inoculation is possible in barley, and this movement may serve to dilute the toxin.

Transport proteins may play an important role in the micromovement of trichothecene mycotoxins within cells and between cells. We identified a set of DON-induced gene transcripts that encode transporters, including five $\mathrm{ABC}$-family transporters (Verrier et al. 2008), all of which exhibited a 20-fold or more increase after DON treatment. Of these transcripts, one encodes a protein belonging to the multidrug resistance (MDR) subfamily that exhibited transcript accumulation only in DONtreated plants (Table 1) and two encode proteins in the MDRassociated protein (MRP) subfamily that exhibited transcript

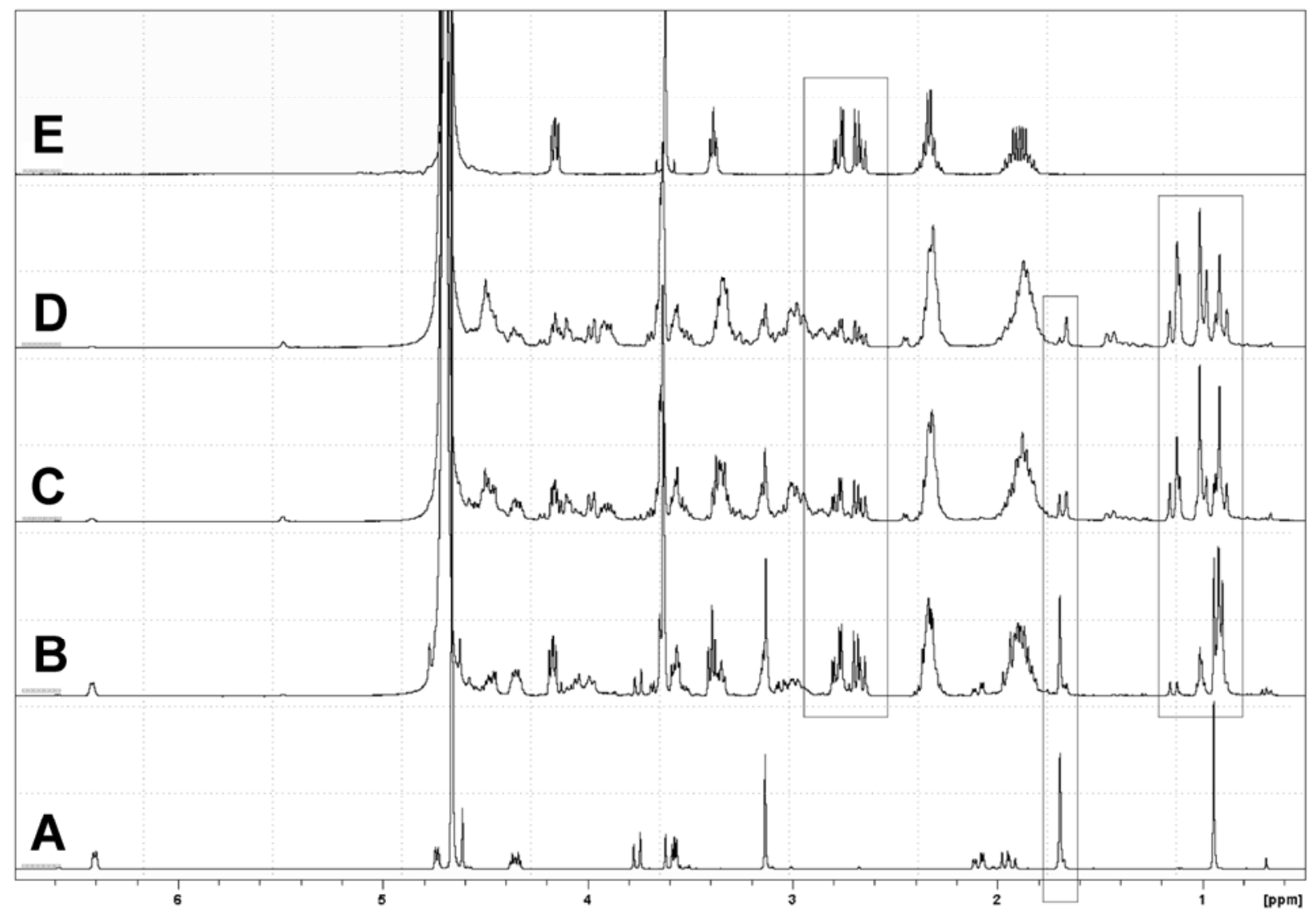

Fig. 6. ${ }^{1} \mathrm{H}$ nuclear magnetic resonance (NMR) spectra of deoxynivalenol (DON) and glutathione (GSH). A, DON only. B, DON plus GSH 3 h after mixing. C, DON plus GSH 1 day after mixing. D, DON plus GSH 3 days after mixing. E, GSH only. Selected peaks indicative of the parental substances and products are boxed. The GSH peak is approximately $2.7 \mathrm{ppm}$. The DON peak is approximately 1.7 . The area rich in product peaks is approximately $1 \mathrm{ppm}$ $(0.9,0.92$, and peaks above $1 \mathrm{ppm}$. Note the single DON peak in this range $[\mathrm{A}])$.

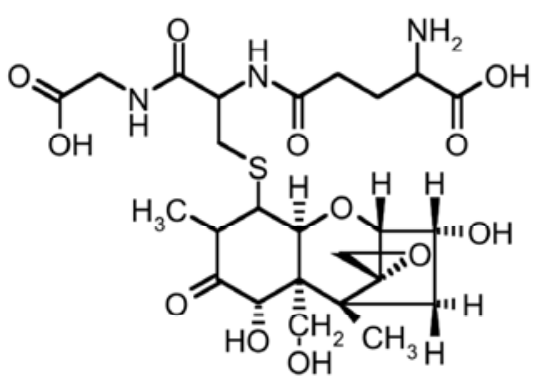

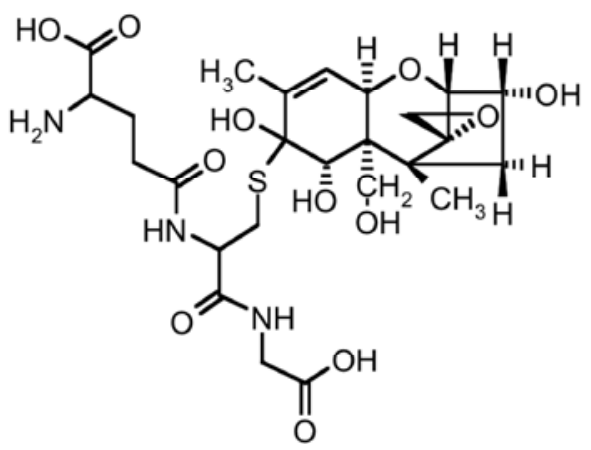

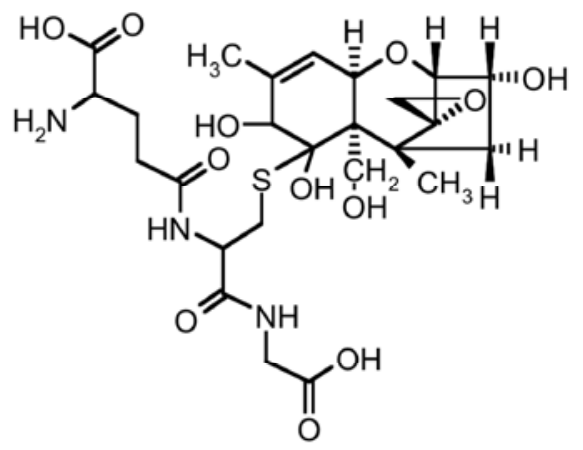

Fig. 7. Structures of possible deoxynivalenol (DON)-glutathione adducts with a mass of 603.65 (sum formula C25H37N3O12S). Left: Adduct to the C=C double bond in DON, middle: adduct at keto group of DON, and right: adduct to keto group of iso-DON. 
accumulation in DON-treated plants and in planta Fusarium spp.-derived trichothecenes (Table 2). In general, ABC proteins have been shown to couple ATP hydrolysis to the transport of substrates across either the plasma membrane or, in the case of MRP, into the vacuole (Rea et al. 1998; Jasinski et al. 2003). We have identified an MRP barley homolog (Contig21298_at) exhibiting a 13.7- to over 180-fold increase in transcript accumulation compared with the water control. Biochemical assays of several Arabidopsis MRP in yeast have demonstrated their role in transfer of GSH-S-conjugates of toxic compounds into the vacuole (Kreuz et al. 1996; Lu et al. 1997, 1998). Also in barley, the ABC-transport associated transfer of a GSH-S-conjugate into the vacuole was previously described (Martinoia et al. 1993; Hinder et al. 1996). In the barley-DON interaction, the movement of DON and DON conjugates from the cytosol into the intercellular space by PDR and MDR proteins or vacuole by MRP proteins may be an effective means of reducing the concentration of toxins in the cytoplasm. Taken together, these results indicate that transporter proteins may play an important role in barley host defense against trichothecene mycotoxins.

DON concentration was also shown to decrease over time as the amount of D3G increased (Fig. 2). The overall pattern of the data shows that a portion of DON in the spike tissue is converted to D3G. From our estimates, a maximum of $21.8 \mathrm{~mol} \%$ of our recovered DON was converted to D3G at 21 days after inoculation. This indicates that an inducible UDP-glucosyltransfere could play an important role in detoxification but that treatment of all spikes with high amounts of DON blocking translation of the induced transcript may delay and reduce the detoxification. From previous studies examining the barley response to in planta trichothecene accumulation, gene transcripts encoding three UDP-glucosyltransferases were identified and classified as trichothecene-specific (Boddu et al. 2007). In our experiment, two UDP-glucosyltransferases with low similarity to the Arabidopsis DOGT1 gene were significantly induced by DON application and in planta-derived trichothecenes, exhibiting 16.5- and 95.9-fold increases in transcript accumulation compared with water (Table 2). UDP-glucosyltransferases are encoded by a very large gene family in plants (Ross et al. 2001; Bowles et al. 2006), with more than 100 genes in Arabidopsis and more than 140 in rice and Brachypodium spp. According to our experience, it is not possible to predict substrate specificity from sequence similarity (Schweiger et al. 2010). Therefore, we have started to functionally test full-length cDNAs of several of the DON and Fusarium spp.-induced candidate UDP-glucosyltransferases by heterologous expression in yeast, and could validate that one gene is conferring DON resistance (Schweiger et al. 2010).

\section{The potential role of GSH in DON resistance.}

We detected two transcripts that encode enzymes catalyzing the final steps in cysteine biosynthesis, annotated as O-acetylserine (thiol)-lyase (Contig23272_at) (Table 1) and "cysteine synthase" (= O-acetylserine (thiol)-lyase, ContigHV12E23u_at) (Table 2). The latter transcript exhibited an increase of 113fold after DON treatment compared with the water control, which is the second highest fold change in our dataset (after Contig21298_at: MRP1, 182-fold). The other transcript encoding a "SATase isoform" (Contig23272_at) was induced over sevenfold. An increased level of cysteine biosynthesis may increase the levels of the cysteine-containing peptides such as GSH, or prevent its depletion, because cysteine is a constituent in the first (rate-limiting) catalytic step in the production of GSH. Plants use O-acetyl(thiol) lyase (= cysteine synthase) to produce cysteine from sulfide and $\mathrm{O}$-acetyl-serine. The enzyme serine-acetyltransferase (SATase) providing the substrate
O-acetyl-serine (SAT) forms a complex with O-acetyl(thiol) lyase (OASTL) that responds in complex allosteric interactions to substrate and end-product concentrations (Hell and Wirtz 2008). In different plants, a huge excess of OASTL over SAT was found (Wirtz and Hell 2006), indicating flux limitation by SAT rather than OASTL. The cysteine biosynthetic enzymes are located in the cytosol, mitochondria, and chloroplasts in plants and are encoded by multiple genes. Thus, a complex interplay of transport processes between the cytosol, mitochondria, and chloroplast may be necessary to allow cysteine retrieval during plant defense (Rausch and Wachter 2005). Evidence from A. thaliana mutants (Haas et al. 2008) and analysis of transgenic plants overexpressing cysteine biosynthetic enzymes (Sirko et al. 2004) indicates that mitochondrial SATase providing O-acetylserine is the pacemaker of cysteine synthesis, and that SATase-overexpressing plants also have increased GSH content.

Our study also detected six GST induced by DON treatment, including a GST24 expressed at 30-fold levels after DON treatment compared with mock water treatment. Interestingly, none of the six GST were identified as preferentially expressed exclusively during DON treatment or during in planta trichothecene accumulation, indicating that these genes respond to a combination of tricothecene accumulation and $F$. graminearum infection. The precise role for GST in ameliorating the effects of DON are unknown.

We also observed that yeast overexpressing a cysteine synthesis gene and grown on medium containing GSH increases DON resistance (data not shown), strongly supporting the hypothesis that GSH-dependent detoxification of DON exists. Using LC-MS/MS and NMR, we obtained evidence that GSH adducts of DON are formed (Figs. 5 and 6). This reaction occurred nonenzymatically, especially at high $\mathrm{pH}$, which does not exclude the possibility that GSH conjugate formation is enzyme catalyzed in vivo. Putative structures of adducts formed with DON and its tautomers (Türker and Gümüs 2009) (e.g., iso-DON) consistent with the NMR results are shown in Figure 7. Potentially, the products with two GSH added may exist. Unfortunately, the adducts found using LC-MS/MS are only stable in the presence of an excess of GSH, thus far preventing their purification and detection in DON-treated plant material. Further research is needed to clarify this issue and to establish assays to test for enzymatic formation of DON-GSH conjugates and the involvement of GST and cysteine synthase genes.

\section{DON drastically induces the expression of cytochrome P450s.}

Proteins involved in metabolic functions are involved in a broad variety of cellular processes, and many genes in this class operate in activities that directly or indirectly promote plant defense. The cytochrome P450 (CYP450) family comprises the largest class of enzymes in plants involved in the biosynthesis of plant hormones and metabolites, and has been shown to be induced in plants exposed to stress or pathogen infection (Ehlting et. al. 2008). Kong and associates (2005) demonstrated the induction of CYP450 genes in F. graminearuminfected resistant and susceptible wheat genotypes. In our study, we detected 36 transcripts involved in metabolic functions, 15 of which were expressed at 20 -fold levels or higher. Of these, we identified nine putative members of the cytochrome CYP450 family, three of which were expressed at approximately 35-fold (Contig20974_at) (Table 2), 50-fold (Contig22479_at) (Table 1), and 60-fold (Ebro08_SQ004_B22_at) (Table 2) in DON-treated compared with the water control-treated plants. Five of the CYP450 gene transcripts exhibited increased transcript accumulation in the DON-treated and in planta derived 
trichothecenes (Table 2), and one of the CYP450 gene transcripts exhibited increased transcript accumulation exclusively in the DON-treated plants (Table 1). No evidence is available for in planta metabolism of DON by hydroxylation or epoxidation reactions typically performed by CYP450 enzymes. A more likely scenario is that synthesis of antifungal metabolites are triggered by DON. An understanding of the role of CYP450 genes may require analysis by advanced metabolomics techniques. In a study comparing genetically unrelated Fusarium-resistant and -susceptible cultivars of wheat, 10 unidentified compounds specific for the resistant line were induced by DON (Paranidharan et al. 2008).

\section{DON induces a barley homolog}

of the Arabidopsis $\mathrm{NF}-\mathrm{X} 1$ gene, a negative regulator of trichothecene-induced defense response.

Trichothecenes appear to act as elicitors triggering a multitude of plant responses (Desmond et al. 2008.). For example, T-2 toxin has elicitor-like activity when infiltrated into the leaves of Arabidopsis (Nishiuchi et al. 2006), inducing expression of the AtNF-XI gene, a homologue of a putative transcription repressor (Masuda et al. 2007). Loss-of-function mutations in the Arabidopsis $N F-X 1$ gene indicated that AtNF-X1 is a negative regulator of trichothecene-induced defense responses (Asano et al. 2007). In our study, we also detected upregulation of transcripts that encode a barley homolog of $N F-X 1$, thus demonstrating that type $B$ trichothecenes are capable of inducing the transcription of this gene.

\section{The role of DON in protein translation.}

Our data provide evidence that DON alone produces highly complex and dynamic effects. At different concentrations of DON, which are changing over time and position from the original source of DON, the ability to translate the induced transcripts into proteins may vary dramatically. Because many transcription factors are induced, it is not possible to distinguish primary and secondary effects (mediated by transcription factors or induced genes affecting plant hormone balance) after $12 \mathrm{~h}$.

The class of genes that show accumulation exclusively after DON application are probably an indication that concentrations as high as those used in the toxin treatment do not occur in tissue challenged with DON-producing Fusarium spp. This class of induced genes could highlight relevant effects and physiological responses that would otherwise be overlooked. After $12 \mathrm{~h}$, genes encoding enolase and lysyl-tRNA synthetase were highly induced (over 33- and 30-fold, respectively) only in DONtreated plants (Table 1). Increased expression of lysyl tRNA synthetase is induced by abiotic stress conditions in several plants and yeast and promotes translational recoding and stop codon readthrough (Wu et al. 2007). Nuclear-encoded tRNA synthetases are required for both cytosolic and organellar protein biosynthesis. In yeast, one aminoacylated tRNA-Lys is imported into mitochondria, although the yeast mitochondrial genome encodes a full complement of tRNA genes (Schneider and MarechalDrouard 2000). In this import process, the lysyl synthetase precursor is required as an import carrier for the tRNA-LYS, and enolase is recruited as a cofactor for import (Entelis et al. 2006). Therefore, the coordinated upregulation of enolase and lysyltRNA synthetase in barley may be of functional relevance. Biochemical evidence for import of a large number of nuclearencoded tRNAs into mitochondria is available for wheat, where they obviously are needed to compensate for the absence of the respective mitochondrial tRNAs (Glover et al. 2001). In wheat, genes for tRNA-LYS exist in both mitochondria and chloroplast (Ogihara et al. 2000, 2005). Due to the presence of a tRNA-LYS gene in wheat mitochondria, the question of whether nuclear-encoded imported tRNA-LYS exists in wheat mitochondria was not addressed in previous studies. Coexistence of nuclear-encoded imported tRNAs and organelle-encoded tRNAs in plants has been described; for instance, in liverwort mitochondria (Akashi et al. 1998). A possible role for redundancy is exemplified in yeast. The mitochondrial-encoded tRNA-LYS needs thiolation in the anticodon (UUU) to allow wobbling to decode both lysine codons and, under elevated temperature, the reduced modification creates a requirement for the import of the nuclearencoded tRNA-LYS (CUU) (Kamenski et al. 2007). More research is needed to test whether biochemical evidence for DONstress- or cysteine-depletion-induced import of tRNA-LYS can be obtained in barley.

In summary, the transcriptome analysis suggests that several modes of action exist in barley that can antagonize the Fusarium spp. virulence factor DON, such as drug efflux, detoxification by glucosylation, and GSH conjugate formation. Also, adaptation of the organellar translation machinery may be more important than previously anticipated. The most important task for the future will be to use the knowledge gained from transcriptomic studies and to validate candidate genes for their relevance and practical utility in Fusarium spp. resistance breeding.

\section{MATERIALS AND METHODS}

Plant materials and growth conditions.

Barley (cv. Morex) seed were sterilized in a 5\% solution of bleach for $15 \mathrm{~min}$ and rinsed. To promote uniform germination, seed were cold/dark treated at $4^{\circ} \mathrm{C}$ for $12 \mathrm{~h}$. Five seeds were planted in Scotts MetroMix 200 in 6-in. standard pots and grown in the growth chamber under the following conditions: $20^{\circ} \mathrm{C}$ for $16 \mathrm{~h}$ with light and $18^{\circ} \mathrm{C}$ for $8 \mathrm{~h}$ in darkness. At 1 week after planting, $5 \mathrm{ml}$ of Osmocote 14/14/14 was added to each pot. Plants were watered as needed until germination and then watered daily until time of inoculation and sampling.

\section{DON treatment, sampling, and experimental design.}

Inoculations were done on the second or third day after the emergence of spikes from the boot (6.05 emergence of inflorescence completed GRO:0007104). DON (CAS: 51481-10-8; Sigma-Aldrich, St. Louis) was dissolved in water before use to a final concentration of $2 \mu \mathrm{g}$ of DON per $10 \mu \mathrm{l}$. Nine florets per spike from the middle portion were inoculated with DON at $2 \mu \mathrm{g} /$ floret or sterile water at $10 \mu \mathrm{l} /$ floret (mock control). The entire spike was then covered in a clear plastic zip bag for up to 72 hai. All inoculations were conducted at 11:00 a.m. Plants were sampled at 1, 6, 12, 24, 48, and 72 hai. Nine florets per spike from a single spike per plant were sampled. In all, 5 to 7 plants/treatment/timepoint were sampled. Six florets immediately below (adjacent to) and six from above the DONinoculated florets were collected as a basipetal and acropetal sample, respectively. Each sample was flash frozen in liquid nitrogen immediately upon sampling and stored at $-80^{\circ} \mathrm{C}$. Three biological replications of this experiment were conducted with a completely randomized block design.

\section{DON analysis.}

Determination of DON concentration in samples was conducted in the mycotoxin lab in the Department of Plant Pathology (University of Minnesota, St. Paul). DON concentration was determined by gas chromatography (GC)-MS following the method described by Mirocha and associates (1998).

\section{Conversion of DON to D3G in planta.}

The barley cv. Morex was selected for the study. All florets on a spike were treated with $2 \mu \mathrm{g}$ of DON between the palea and lemma. Mock controls were treated with water. Four 
spikes per treatment were sampled at $1,3,5,7,10,14$, and 21 days after inoculation. Three biological replications of this experiment were conducted. Sampled spikes were ground into powder in liquid nitrogen and $10 \mathrm{~g}$ of each sample was extracted in acetonitrile/water mixed at a ratio of $84: 16(\mathrm{vol} / \mathrm{vol})$ by shaking at $180 \mathrm{rpm}$ for $90 \mathrm{~min}$. The extracts were filtered and pressed through MycoSep 230 Trich columns (Romer Lab Inc., Union, MO, U.S.A.). The filtrate $(4 \mathrm{ml})$ was dried in a speed vac and the residue was dissolved in $1 \mathrm{ml}$ of methanol/water mixture (10:90, vol/vol). This sample was passed through 0.45-m filters prior to LC-MS/MS analysis (Berthiller et al. 2005).

\section{RNA extraction and}

Barley1 Affymetrix GeneChip probe-array hybridization.

RNA was isolated from DON and water-treated florets sampled at 1, 12, 24, and 48 hai. Three biological replications were conducted. The samples were ground in liquid nitrogen. Total RNA was extracted from $0.5 \mathrm{~g}$ of tissue according to the Trizol (Invitrogen, Carlsbad, CA, U.S.A.) protocol, then subjected to treatment with RQ1 RNase-free DNase (Promega Corp., Madison, WI, U.S.A.) and purified in RNeasy Mini columns (Qiagen, Valencia, CA, U.S.A.). Purified samples were quality checked by microelectrophoresis using the Agilent 2100 Bioanalyzer system (Agilent, Santa Clara, CA, U.S.A.). Samples were then submitted to the Biomedical Genomics Center, University of Minnesota, Minneapolis for labeling and hybridization according to procedures outlined by Boddu and associates (2006). The Barley1 GeneChip (Affymetrix, Santa Clara, CA, U.S.A.) represents approximately 22,439 genes with 22,792 probe sets (Close et al. 2004). Array content was derived from expressed sequence tags from 84 barley cDNA libraries representing several genotypes, developmental stages, and biotic (Fusarium, Pyrenophora, and Blumeria treated) and abiotic (light, waterlogged or drought, cold, salt, and nitrogen) treatments.

\section{GeneChip data analysis.}

GeneChip data were collected from barley spikes at 1, 12, 24 , and $48 \mathrm{~h}$ after DON and water inoculation and analyzed using GeneChip Expressionist Pro. The data were first normalized using robust multi-chip analysis with the Refiner module in GeneChip Expressionist. N-way analysis of variance was conducted to test for significance using the Analyst module. Gene transcripts that exhibited differential accumulation $(P<$ 0.0001 ; twofold difference) between the DON- and waterinoculated plants were selected at each of the timepoints. $\mathrm{BH}$ Q values (corrected $P$ values) (Benjamini and Hochberg 1995) were computed at an FDR of 5\%. Correlation values between replications ranged from 97 to $99 \%$. All GeneChip data (CEL and DAT) can be found in the BarleyBase database (accession number BB62). Functional annotations of gene transcripts were derived from the HarvEST Barley Database 1.63. All annotations were associated with an $e$ value of $1 \mathrm{E}-10$ or smaller.

\section{qRT-PCR experimental design and analysis.}

We selected a subset of candidate transcripts from the Barley $1 G$ eneChip dataset based on fold-change values indicative of a toxin-specific response. These transcripts encoded the following: senescence-associated protein (Contig14507_at), NFX1 transcription factor (Contig 7127_at), F-box protein (HD08H417r_at), cytochrome P450 protein (Contig 22479_at), UDP-glucosyltransferase (Contig13248_at), and gibberellin receptor GIDIL2 (Contig20431_at). We designed primers (Supplementary Table 5) with Primer Express Software, according to parameters standard for real-time PCR assays (BioRad Laboratories, Hercules, CA, U.S.A.). Standard RT-PCR using SuperScript One-Step RT-PCR System with Platinum
Taq DNA polymerase was used to verify the compatibility of all primer sets (Invitrogen). PCR products were purified and sequenced for target specificity at the Biomedical Genomics Center (University of Minnesota, St. Paul, MN, U.S.A.).

Real-time RT-PCR of candidate genes was carried out with the Chromo 4 Real-Time System using 96-opaque-well realtime RT-PCR optimized plates sealed with Optical Microseal Adhesives (Bio-Rad Laboratories). We measured transcript accumulation of the target gene and the positive control gene, barley $\alpha$-tubulin, within all samples (DON or water treatment, 1 and 12 hai) of a single replication on each 96-well plate. Each treatment-timepoint-genotype combination was technically replicated three times. Six negative control wells on each plate contained the reaction mixture, with water as a mock template, three of which contained primer sequences for the target gene and three containing primers for the gene of reference. The experiment was repeated with a total of three biological replications of treated samples. We used MJ Opticon Monitor Software, version 3.1, for visualizing and analyzing real-time PCR data (Bio-Rad Laboratories). Raw cycle threshold $(\mathrm{Ct})$ values for each timepoint-treatment-replication combination of each transcript were exported into Microsoft Excel. Technical replications were averaged for each sample, and then mean $\mathrm{Ct}$ values were calculated by averaging the biological replications. The $\Delta \Delta$ comparisons were calculated for each transcript as follows: $\left(\Delta \mathrm{Ct}_{\text {target }}-\Delta \mathrm{Ct}_{\text {reference }}\right)=\left(\mathrm{Ct}_{\text {target, } \mathrm{H} 2 \mathrm{O}}-\right.$ $\left.\mathrm{Ct}_{\text {target, DON }}\right)-\left(\mathrm{Ct}_{\text {reference, H2O }}-\mathrm{Ct}_{\text {reference, DON }}\right)$. Tests of significance based on these values were determined between treatments (DON and water). For the treatment comparisons, $\Delta \Delta$ values for water samples were assigned a baseline value of 0 . Pfaffl (2006) outlined the basic procedures of relative quantification we used in this experiment.

\section{LC-MS analysis.}

LC-MS analysis was performed using an 1100 Series HPLC (Agilent, Waldbronn, Germany) coupled to a QTrap mass spectrometer (Applied Biosystems, Foster City, CA, U.S.A.). Separation was performed on a Phenomenex Gemini C18 column (150 by $4.6 \mathrm{~mm}, 5 \mu \mathrm{m}$ ) using a linear gradient from 5 to $97 \%$ aqueous methanol containing $5 \mathrm{mM}$ ammonium acetate and $1 \%$ acetic acid at $25^{\circ} \mathrm{C}$. Then, $2 \mu \mathrm{l}$ of the $1: 50$ diluted samples also used for NMR analysis was injected. Ionization was performed using a Heated Nebulizer (APCI) source in negative ion mode at $450^{\circ} \mathrm{C}$ with a needle current of $-2 \mu \mathrm{A}$. Enhanced mass scans from 250 to $1,000 \mathrm{amu}$ were acquired, with a scan rate of 1,000 $\mathrm{amu} / \mathrm{s}$ using dynamic fill time for the iontrap. Gas settings were as follows: curtain gas, $20 \mathrm{psi}(138 \mathrm{kPa})$; nebulizer gas, $60 \mathrm{psi}$ (414 kPa); and auxiliary gas, 15 psi $(103 \mathrm{kPa})$.

\section{NMR measurements.}

For the NMR spectroscopic investigation, a sample was prepared by dissolving approximately $20 \mathrm{mg}$ of DON and $40 \mathrm{mg}$ of $\mathrm{GSH}$ in $1 \mathrm{ml}$ of $\mathrm{D}_{2} \mathrm{O}$ and adjusting the $\mathrm{pH}$ to 8 with concentrated aqueous ammonia. A 5-mm NMR tube was charged with $0.7 \mathrm{ml}$ of this solution and sealed. ${ }^{1} \mathrm{H},{ }^{13} \mathrm{C},{ }^{1} \mathrm{H}^{1} \mathrm{H} \operatorname{COSY},{ }^{1} \mathrm{H}^{13} \mathrm{C}$ HSQC, and ${ }^{1} \mathrm{H}^{13} \mathrm{C}$ HMBC spectra were recorded on a Bruker Avance DRX-400 FT-NMR spectrometer using standard pulse programs from the Bruker software library.

\section{ACKNOWLEDGMENTS}

This work was supported by grants to G. J. Muehlbauer from the U.S. Wheat and Barley Scab Initiative (United States Department of Agriculture-Agricultural Research Service) and the Minnesota Small Grains Initiative, and by grants from the Austrian Science Fund FWF to G. Adam (SFB F37 and L475-B09). Part of the work of G. Adam was also funded by the EU FP5 project FUCOMYR. We thank the Biomedical Image Processing Facility at the University of Minnesota for the GeneChip labeling 
and hybridization, Y. Dong for technical support in conducting the DON concentration analysis, W. Xu and Z. Tu at the University of Minnesota Supercomputing Institute for bioinformatics assistance, G. Wiesenberger for critically reading the manuscript, and $\mathrm{H}$. Weindorfer for technical assistance.

\section{LITERATURE CITED}

Akashi, K., Takenaka, M., Yamaoka, S., Suyama, Y., Fukuzawa, H., and Ohyama, K. 1998. Coexistence of nuclear DNA-encoded valine tRNA(AAC) and mitochondrial DNA-encoded valine tRNA(UAC) in mitochondria of a liverwort Marchantia polymorpha. Nucleic Acids Res. 26:2168-2172.

Asano, T., Masuda, D., Yasuda, M., Nakashita, H., Kudo, T., Kimura, M. Yamaguchi, K., and Nishiuchi, T. 2007. AtNFXL1, an Arabidopsis homologue of the human transcription factor NF-X1, functions as a negative regulator of the trichothecene phytotoxin-induced defense response. Plant J. 53:450-464.

Bai, G., and Shaner, G. 2004. Management and resistance in wheat and barley to Fusarium head blight. Annu. Rev. Phytopathol. 42:135-161.

Bai, G. H., Desjardins, A. E., and Plattner, R. D. 2001. Deoxynivalenolnonproducing Fusarium graminearum causes initial infection, but does not cause disease spread in wheat spikes. Mycopathologia 153:91-98.

Benjamini, Y., and Hochberg, Y. 1995. Controlling the false discovery rate: A practical and powerful approach to multiple testing. J. R. Stat. Soc. B. 57:289-300

Berthiller, F., Dall'Asta, C., Schuhmacher, R., Lemmens, M., Adam, G., and Krska, R. 2005. Masked mycotoxins: Determination of a deoxynivalenol glucoside in artificially and naturally contaminated wheat by liquid chromatography-tandem mass spectrometry. J. Agric. Food. Chem. 9:3421-3425.

Berthiller, F., Corradini, R. Dall'Asta, C., Marchelli, R., Sulyok, M., Krska, R., Adam, G., and Schuhmacher, R.. 2009. Occurrence of deoxynivalenol and its 3- $\beta$-D-glucoside in wheat and maize. Food Addit. Contam. A 26:507-511.

Boddu, J., Cho, S., Kruger, W. M., and Muehlbauer, G. J. 2006. Transcriptome analysis of the barley-Fusarium graminearum interaction. Mol. Plant-Microbe Interact. 19:407-417.

Boddu, J., Cho, S., and Muehlbauer, G. J. 2007. Transcriptome analysis of trichothecene-induced gene expression in barley. Mol. Plant-Microbe Interact. 20:1364-1375.

Bowles, D., Lim, E., Poppenberger, B., and Vaistij, F. E. 2006. Glycosyltransferases of lipophilic small molecules. Annu. Rev. Plant Biol. 57:567-597.

Close, T. A., Wanamaker, S., Caldo, R. A., Turner, S. M., Ashlock, D. A., Dickerson, J. A., Wing, R. A., Muehlbauer, G. J., Kleinhofs, A., and Wise, R. P. 2004. Global expression profiling in cereals: 22K barley GeneChip comes of age. Plant Physiol. 134:960-968

de la Peña, R. C., Smith, K. P., Capettini, F., Muehlbauer, G. J., GalloMeagher, M., Dill-Macky, R., Somers, D. A., and Rasmusson, D. C. 1999. Quantitative trait loci associated with resistance to Fusarium head blight and kernel discoloration in barley. Theor. Appl. Genet. 99:561569

Desjardins, A. E., Hohn, T. M., and McCormick, S. P. 1993. Trichothecene biosynthesis in Fusarium species: Chemistry, genetics, and significance. Microbiol. Rev. 57:595-604.

Desjardins, A. E., Proctor, R. H., Bai, G., McCormick, S. P., Shaner, G., Buechley, G., and Hohn, T. 1996. Reduced virulence of trichothecene non-producing mutants of Gibberella zeae in wheat field tests. Mol. Plant-Microbe Interact. 9:775-781.

Desjardins, A. E., Bai, G., Plattner, R. D., and Proctor, R. H. 2000. Analysis of aberrant virulence of Gibberella zeae following transformation mediated complementation of a trichothecene-deficient (Tri5) mutant. Microbiology 146:2059-2068.

Desmond, O. J., Manners, J. M., Stephens, A. E., Maclean, D. J., Schenk, P. M., Gardiner, D. M., Munn, A. L., and Kazan, K. 2008. The Fusarium mycotoxin deoxynivalenol elicits hydrogen peroxide production, programmed cell death and defence response in wheat. Mol. Plant. Pathol. 9:435-445.

Edwards, R., Dixon, D. P., and Walbot, V. 2000. Plant glutathione $S$-transferases: Enzymes with multiple functions in sickness and in health. Trends Plant Sci. 5:193-198.

Ehlting, J., Sauveplane, V., Olry, A., Ginglinger, J.-F., Provart, N. J., and Werck-Reichhart, D. 2008. An extensive (co-)expression analysis tool for the cytochrome P450 superfamily in Arabidopsis thaliana. BMC Plant Biol. 8:47.

Entelis, N., Brandina, I. Kamenski, P., Krasheninnikov, I. A., Martin, R. P., and Tarassov, I. 2006. A glycolytic enzyme, enolase, is recruited as a cofactor of tRNA targeting toward mitochondria in Saccharomyces cerevisiae. Genes Dev. 20:1609-1620.

Espinet, C., de la Torre, M. A., Aldea, M., and Herrero, E. 1995. An efficient method to isolate yeast genes causing overexpression-mediated growth arrest. Yeast 11:25-32.

Glover, K. E., Spencer, D. F., and Gray, M. W. 2001. Identification and structural characterization of nucleus-encoded transfer RNAs imported into wheat mitochondria. J. Biol. Chem. 276:639-48.

Grove, J. F. 2007. The trichothecenes and their biosynthesis. Prog. Chem Org. Nat. Prod. 88:63-130.

Haas, F. H., Heeg, C., Queiroz, R., Bauer, A., Wirtz, M., and Hell, R. 2008. Mitochondrial serine acetyltransferase functions as a pacemaker of cysteine synthesis in plant cells. Plant Physiol. 148:1055-1067.

Hell, R., and Wirtz. M. 2008. Metabolism of cysteine in plants and phototrophic bacteria. Pages 59-91 in: Sulfur Metabolism in Phototrophic Organisms: Advances in Photosynthesis and Respiration Vol 27. R. Hell, C. Dahl, D. Knaff, and T. Leustek, eds. Springer, Dordrecht, The Netherlands.

Hinder, B., Schellenberg, M., Rodoni, S., Ginsburg, S., Vogt, E., Martinoia, E., Matile, P., and Hörtensteiner, S. 1996. How plants dispose of chlorophyll catabolites. J. Biol. Chem. 271:27233-27236.

Jasinski, M., Ducos, E., Martinoia, E., and Boutry, M.. 2003. The ATPbinding cassette transporters: Structure, function and gene family comparison between rice and Arabidopsis. Plant Physiol. 131:1169-1177.

Kamenski, P., Kolesnikova, O., Jubenot, V., Entelis, N., Krasheninnikov, I. A., Martin, R. P., and Tarassov, I. 2007. Evidence for an adaptation mechanism of mitochondrial translation via tRNA import from the cytosol. Mol. Cell 26:625-637.

Kang, Z., and Buchenauer, H. 1999. Immunocytochemical localization of Fusarium toxins in infected wheat spikes by Fusarium culmorum. Physiol. Mol. Plant Pathol. 55:275-288.

Kong, L., Anderson, J. M., and Ohm, H. W. 2005. Induction of wheat defense and stress-related genes in response to Fusarium graminearum Genome 48:29-40.

Kreuz, K., Tommasini, R., and Martinoia, E. 1996. Old enzymes for a new job: Herbicide detoxification in plants. Plant Physiol. 111:349-353

Lemmens, M., Scholz, U., Berthiller, F., Dall'Asta, C., Koutnik, A., Schuhmacher, R., Adam G., Buerstmayr, H., Mesterhazy, A., Krska, R. and Ruckenbauer, P. 2005. The ability to detoxify the mycotoxin deoxynivalenol colocalizes with a major quantitative trait locus for Fusarium head blight resistance in wheat. Mol. Plant-Microbe Interact. 18:1318-1324.

Liu, S., Zhang, X., Pumphrey, M. O., Stack, R. W., Gill, B. S., and Anderson, J. A. 2006. Complex microcolinearity among wheat, rice, and barley revealed by fine mapping of the genomic region harboring a major QTL for resistance to Fusarium head blight in wheat. Funct. Intgr. Genomics 6:83-89.

Lu, Y. P., Li, Z. S., and Rea, P. A. 1997. AtMRP1 gene of Arabidopsis encodes a glutathione $S$-conjugate pump: Isolation and functional definition of a plant ATP-binding cassette transporter gene. Proc. Natl. Acad. Sci. U.S.A. 94:8243-8248

Lu, Y. P., Li, Z. S., Drozdowicz, Y. M., Hortensteiner, S., Martinoia, E. and Rea, P. A. 1998. AtMRP2, an Arabidopsis ATP binding cassette transporter able to transport glutathione $S$-conjugates and chlorophyll catabolites: Functional comparisons with Atmrp1. Plant Cell 10:267282

Lulin, M., Yi, S., Aizhong, C., Zengjun, Q., Liping, X., Peidu, C., Dajun, L., and Xiu-e, W. Molecular cloning and characterization of an up-regulated UDP-glucosyltransferase gene induced by DON from Triticum aestivum L. cv. Wangshuibai. Mol. Biol. Rep. 37:785-795.

Maier, F. J., Miedaner, T., Hadeler, B., Felk, A., Salomon, S., Lemmens, M., Kassner, H., and Schäfer, W. 2006. Involvement of trichothecenes in fusarioses of wheat, barley, and maize evaluated by gene disruption of the trichodiene synthase (Tri5) gene in three field isolates of different chemotype and aggressiveness. Mol. Plant Pathol. 7:449-461.

Martinoia, E., Grill, E., Tommasini, R., Kreuz, K., and Amrhein, N. 1993. ATP-dependent glutathione $S$-conjugate 'export' pump in the vacuolar membrane of plants. Nature 364:247-249.

Masuda, D., Ishida, M., Yamaguchi, K., Yamaguchi, I., Kimura, M., and Nishiuchi, T. 2007. Phytotoxic effects of trichothecenes on the growth and morphology of Arabidopsis thaliana. J. Exp. Bot. 58:1617-1626.

Mirocha, C. J., Kolaczkowski, E., Xie, W., Yu, H., and Jelen, H. 1998 Analysis of deoxynivalenol and its derivatives (batch and single kernel) using gas chromatography/mass spectrometry. J. Agric. Food Chem. 46:1414-1418

Nduulu, L. M., Mesfin, A., Muehlbauer, G. J., and Smith K. P. 2007. Analysis of the chromosome $2(2 \mathrm{H})$ region of barley associated with the correlated traits Fusarium head blight resistance and heading date. Theor. Appl. Genet. 115:561-570.

Nishiuchi, T. D., Masuda, D., Nakashita, H., Ichimura, K., Shinozaki, K. 
Yoshida, S., Kimura, M., Yamaguchi, I., and Yamaguchi, K. 2006. Fusarium phytotoxin trichothecenes have an elicitor-like activity in Arabidopsis thaliana, but the activity differed significantly among their molecular species. Mol. Plant-Microbe Interact. 19:512-520.

Ogihara, Y., Isono, K., Kojima, T., Endo, A., Hanaoka, M., Shina, T., Terachi, T., Utsugi, S., Murata, M., Mori, N., Takumi, S., Ikeo, K., Gojobori, T., Murai, R., Murai, K., Matsuoka, Y., Ohnishi, Y., Tajiri, H., and Tsunewaki, K. 2000. Chinese spring wheat (Triticum aestivum L.) chloroplast genome: Complete sequence and contig clones. Plant Mol. Biol. Rep. 18:243-253.

Ogihara, Y., Yamazaki, Y., Murai, K., Kanno, A., Terachi, T., Shiina, T., Miyashita, N., Nasuda, S., Nakamura, C., Mori, N., Takumi, S., Murata, M., Futo, S., and Tsunewaki, K. 2005. Structural dynamics of cereal mitochondrial genomes as revealed by complete nucleotide sequencing of the wheat mitochondrial genome. Nucleic Acids Res. 33:6235-6250.

Paranidharan, V., Abu-Nada, Y., Hamzehzarghani, H., Kushalappa, A. C., Mamer, O., Dion, Y., Rioux, S., Comeau, A., and Choiniere, L. 2008. Resistance-related metabolites in wheat against Fusarium graminearum and the virulence factor deoxynivalenol (DON). Can. J. Bot. 86:11681179.

Pestka, J. J., and Smolinski, A. T. 2005. Deoxynivalenol: Toxicology and potential effects on humans. J. Toxicol. Environ. Health B Crit. Rev. 8:39-69.

Pfaffl, M. W. 2006. Relative quantification. Pages 63-82 in: Real-Time PCR. T. Dorak, ed. Taylor \& Francis Publishers, New York.

Poppenberger, B., Berthiller, F., Lucyshyn, D., Sieberer, T., Schuhmacher, R., Krska, R., Kuchler, K., Glössl, J., Luschnig, C., and Adam. G. 2003. Detoxification of the Fusarium mycotoxin deoxynivalenol by a UDPglucosyltransferase from Arabidopsis thaliana. J. Biol. Chem. 278:47905-47914.

Proctor, R. H., Hohn, T. M., and McCormick, S. P. 1995. Reduced virulence of Gibberella zeae caused by disruption of a trichothecene toxin biosynthetic gene. Mol. Plant-Microbe Interact. 4:593-601.

Rausch, T., and Wachter, A. 2005. Sulfur metabolism: A versatile platform for launching defence operations. Trends Plant Sci. 10:503-509.

Rea, P. A., Li, Z.-S., Lu, Y.-P., Drozdowicz, Y. M., and Martinoia, E. 1998. From vacuolar GS-X pumps to multispecific ABC transporters. Ann. Rev. Plant Physiol. Plant Mol. Biol. 49:727-760.
Ross, J., Li, Y., Lim, E., and Bowles, D. J. 2001. Higher plant glycosyltransferases. Genome Biol. (Rev.) 2:3004.1-3004.6

Savard, M. E., Sinha, R. C., Seaman, W. L., and Fedak, G. 2000. Sequential distribution of the mycotoxin deoxynivalenol in wheat spikes after inoculation with Fusarium graminearum. Can. J. Plant Pathol. 22:280285.

Schneider, A, and Marechal-Drouard, L. 2000. Mitochondrial tRNA import: Are there distinct mechanisms? Trends Plant Sci. 10:509-513.

Schweiger, W., Boddu, J., Shin, S., Poppenberger, B., Berthiller, F., Lemmens, M., Muehlbauer, G. J., and Adam, G. 2010. Validation of a candidate deoxynivalenol-inactivating UDP-glucosyltransferase from barley by heterologous expression in yeast. Mol. Plant-Microbe Interact. 23:977-986.

Sirko, A., Błaszczyk, A., and Liszewska, F. 2004. Overproduction of SAT and/or OASTL in transgenic plants: a survey of effects. J. Exp. Bot. 55:1881-1888

Türker, L., and Gümüs, S. 2009. A theoretical study on vomitoxin and its tautomers. J. Hazard. Mater. 163:285-294.

Verrier, P. J., Bird, D., Burla, B., Dassa, E., Forestier, C., Geisler, M., Klein, M., Kolukisaoglu U., Lee, Y., Martinoia, E., Murphy, A., Rea, P. A., Samuels, L., Schulz, B., Spalding, E. J., Yazaki, K., and Theodoulou, F. L. 2008. Plant ABC proteins-a unified nomenclature and updated inventory. Trends Plant Sci. 13:151-159.

Waldron, B. L., Moreno-Sevilla, B., Anderson, J. A., Stack, R. W., and Frohberg, R. C. 1999. RFLP mapping of QTL for Fusarium head blight resistance in wheat. Crop Sci. 39:805-811.

Walter, S., Brennan, J. M., Arunachalam, C., Ansari, K. I., Hu, X., Khan, M. R., Trognitz, F., Trognitz, B., Leonard, G., Egan, D., and Doohan, F. M. 2008. Components of the gene network associated with genotypedependent response of wheat to the Fusarium mycotoxin deoxynivalenol. Funct. Integr. Genomics 8:421-427.

Wirtz, M., and Hell, R. 2006. Functional analysis of the cysteine synthase protein complex from plants: Structural, biochemical and regulatory properties. J. Plant Physiol. 163:273-286.

Wu, X. R., Kenzior, A., Willmot, D., Scanlon, S., Chen, Z., Topin, A., He, S. H., Acevedo, A., and Folk, W. R. 2007. Altered expression of plant lysyl tRNA synthetase promotes tRNA misacylation and translational recoding of lysine. Plant J. 50:627-636. 